\title{
Corporate Venture Capital and the Nature of Innovation*
}

\author{
Hannes Maxin ${ }^{\dagger}$ \\ Hannover Economic Papers (HEP) No. 603
}

August 1, 2017

\begin{abstract}
This paper investigates a model where two corporate venture capital firms (CVCs) decide whether to finance a new venture stand-alone or together, called syndication. The CVCs obtain a cash flow if the venture succeeds. In addition, the venture has a positive or negative effect on an asset of the CVCs parental companies. This effect may differ among the parental companies. I show that the CVC faced with the weaker positive effect becomes the stand-alone investor only if the expected cash flow is low. Otherwise, in equilibrium, there are only syndicates or stand-alone investments of the CVC with the stronger positive effect. However, if one CVC faces a positive effect on its parental company's asset whereby the opponent faces a negative effect, then a syndicate is still possible. The model generates empirical predictions for syndicates consisting of several CVCs.
\end{abstract}

Keywords: Corporate Venture Capital; Venture Capital; Nonmonetary Support; Nature of Innovation

JEL classification: G24; M13

*I would like to thank my advisor Heidrun C. Hoppe-Wewetzer for very helpful comments and suggestions. The paper has also benefited from the comments of Thomas Hellmann and the seminar of Leibniz University of Hannover.

$\dagger$ Department of Economics, University of Hannover, Königsworther Platz 1, 30167 Hannover, Germany, E-mail: maxin@mik.uni-hannover.de. 


\section{Introduction}

Corporate venture capital plays an important role in financing young firms with uncertain but high growth expectations. I define corporate venture capital as minority equity investments by an incumbent company in entrepreneurial firms, similar to Dushnitsky (2012). After passing cyclical investment waves in the last decades, National Venture Capital Association (2016) states that nearly $22 \%$ of all venture capital deals are realized by incumbent companies nowadays. ${ }^{1}$

The incumbents frequently use specialized subsidiaries that allocate their corporate venture capital towards young firms. A typical feature of these corporate venture capital firms (CVCs) is that they pursue two different goals: beside high financial returns (e.g. initial public offering or the sale of ownership stakes), there are often more diverse and complex innovation objectives (e.g. an access to new products, a window on new technologies or generating demand). ${ }^{2}$ Hence, corporate venture capital can be seen as an access to otherwise untapped innovations that are critical to the incumbents success and longevity.

By contrast, independent venture capital firms (IVCs) are only driven by financial returns due to the absence of a parental company. As it is well known, both investor types often share the financing cost and the nonmonetary support with other investors. This so-called syndication means in a restrictive sense that a cooperation of two or more investors takes place in a particular financing round. If the term is used more broadly, it also describes situations where investors enter different financing rounds. The former definition can be seen as basis for this paper.

Empirical observations suggest a lack of research about the determinants of syndicates consisting of several CVCs. Park and Steensma (2012) and Souitaris and Zerbinati (2014) state that syndicates between several CVCs are rare. The subsidiaries forgo to syndicate with a competitor's investor due to a possible innovation advantage for their parental company. In line with the above literature, Ivanov and Xie (2010) remark that "CVCs may prevent their portfolio companies from forming alliances with their parent corporations' competitors even though such alliances can bring significant [..] benefits to the start-ups." (p. 133). By contrast, Sharifzadeh and Walz (2012) illustrate that syndicates between several CVCs are possible. NWZ Digital, PDV Inter-Media Venture and Müller Medien, for instance, finance together

\footnotetext{
${ }^{1}$ For a detailed description of the historical background see, for instance, Dushnitsky (2008) and Dushnitsky (2012).

${ }^{2}$ See, for instance Winters and Murfin (1988), McNally (1997), Riyanto and Schwienbacher (2006), Benson and Ziedonis (2009).
} 
the venture AX Semantics.

To study this issue, I consider a model where two CVCs have to decide whether to finance a venture as a stand-alone investor or to syndicate with each other. The critical points are the innovation objectives of the corporate investors. I assume that the success of the venture affects the values of some assets (e.g. products or processes) owned by the parental companies. This change in the assets' values may be positive or negative. Hence, the model has the following two polar cases for the nature of innovation: the venture can be a complement (positive nature) or a substitute (negative nature) for the parental company. ${ }^{3}$ In the model, the change in the assets' values may vary among the incumbents. Thus, the venture can be a weak complement for a parental company, while it is also a strong complement for the other. Moreover, the change in the asset's value may be viewed as a positive external effect if one CVC is the stand-alone investor, whereas the opponent forgoes an investment. On the other hand, if the CVCs found a syndicate, then both investors bear the investment costs.

To my knowledge, no other theoretical paper considers such a financing situation with two CVCs. In this regard, I formulate the following research question: what impact does the young firm's nature of innovation has on the investment decision of two CVCs?

The main results are the following. First, if the venture is a complement for both CVCs, then the CVC with the weaker complement may become the stand-alone investor only if the venture leads to a low expected cash flow. ${ }^{4}$ If the expected cash flow reaches a medium level, only syndicates between the corporate investors occur. For a higher expected cash flow, there are solely syndicates between both investors and stand-alone investments of the CVC with the stronger complement. Second, if an investor is confronted with a substitute and the other investor faces a complement, syndicates between both investors are still possible. Third, the change in the asset's value occurs as a positive external effect for one CVC, if the other CVC is the stand-alone investor. However, the allocation of the shares in a syndicate can balance the change in the assets' values among the CVCs. As a consequence, the shareholding provides an opportunity to internalize this externality, such that both CVCs accept to syndicate. The syndicate enables a higher utility for both CVCs than in the former stand-alone investment situation.

\footnotetext{
${ }^{3}$ Gompers and Lerner (2000), Park and Steensma (2012) and Dushnitsky (2012) state that corporate venture capital is used to finance complementary ventures. However, Masulis and Nahata (2009) show with their sample, that substitutes are corporate-backed as well.

${ }^{4}$ For simplification, I write that the CVCs obtain the utility of an investment and not the particular parental company.
} 
An investment situation involving only CVCs may occur because, in contrast to other investor types like independent venture capital firms (IVCs), corporate investors have the ability to support the young firms better by using certain resources. ${ }^{5}$ Park and Steensma (2012), for instance, stress that the mobile broadband service provider Airvana succeeds because of the CVC's cost-intensive testing infrastructure owned by the parental company. Emphasizing the importance of the support, Chemmanur and Loutskina (2008) also remark that CVCs provide specialized industry expertise to enable a successful development of their ventures. For instance, young FinTech firms are confronted with comprehensive regulatory conditions (e.g. bank licenses or deposit guarantee), so that an adequate support by an incumbent company or its CVC is desirable.

This paper is closely related to Hellmann (2002). An important difference is that I focus on the investment decision of two CVCs, whereas Hellmann (2002) investigates the choice of the venture between an IVC and a CVC. Hence, I consider the change in the asset's value for each corporate investor. Moreover, I state that the valueadded of a syndicate is an important factor. Consequently, I allow all syndication partners to provide nonmonetary support to the venture, in contrast to Hellmann (2002).

Syndicates consisting solely of IVCs are widely observed: Brander et al. (2002) analyze different theoretical hypotheses that offer rational for syndicates of this investor type. Their empirical study favoring the value-added hypotheses, which suggests that syndicates lead to higher expected cash flows than stand-alone investments due to the different nonmonetary support of several financiers. ${ }^{6}$ Tian (2011) examines venture capital syndicates from the point of view of the ventures. Compared to stand-alone investments, Tian (2011) shows that syndicates create a product market value and a financial market value for the ventures. Moreover, Casamatta and Haritchabalet (2007) and Cestone et al. (2006) formally show that an additionally screening advise on a potential venture is the reason for a syndicate. In this way, they follow the selection hypothesis of Lerner (1994).

The syndication decision of IVCs that are confronted with a CVC as syndication partner is only partially analyzed. Hill et al. (2009) state that syndicates between corporate investors and IVCs lead to a higher investment output per year and a

\footnotetext{
${ }^{5}$ See, for instance Block and MacMillan (1993), Maula (2001), Dushnitsky (2008) and Ivanov and Xie (2010).

${ }^{6}$ Sharifzadeh and Walz (2012) remark six reasons for syndication: risk-sharing (Brander et al., 2002), selection (Sah and Stiglitz, 1986; Lerner, 1994), value added (Brander et al., 2002), steady deal flow (Hochberg et al., 2007), window dressing (Lerner, 1994) and staged financing problems (Fluck et al., 2005).
} 
lower closure rate among ventures. In this regard, Hellmann (2002) provides the first explicit model and examines the entrepreneur's choice between a CVC and an IVCs. Hellmann (2002) supposes competition for both the valuation and the nonmonetary support. Similar to the present model, Hellmann (2002) stresses that corporate venture capital investments depend strongly on the nature of innovation of the entrepreneur's product. As a result, the analysis shows that if the young firm is a complement to the large company, the CVC is chosen by the entrepreneur. On the other hand, the IVC is the optimal choice if the young firm is a weak substitute. Syndication is optimal, such that the CVC cooperates with the IVC, only if the young firm is a strong substitute.

The remainder of the paper is organized as follows: in the next section I introduce the theoretical model. Section 3 presents the analysis of the investment decision of both CVCs. In Section 4, I derive empirical predictions from the theoretical results and review existing empirical evidence. The last section concludes. All proofs are included in the appendix.

\section{The Model}

I consider two risk-neutral CVCs, $C V C_{i}$ with $i \in\{1,2\}$, and a wealthless venture which needs a capitalization normalized to 1 . There are two possible future states of nature that I call success and failure. If $C V C_{i}$ decides to finance the new venture and it succeeds, then $C V C_{i}$ obtains the cash flow $R>1$. If the venture fails, there are no cash flows. Success occurs with probability $q \in(0,1)$. If $C V C_{i}$ does not finance the venture, then it obtains a risk-free cash flow $r$, where $1 \leq r<R$.

In order to highlight the innovation objectives of CVCs, I suppose that a particular asset of the parental company is affected by the success of the venture, following Hellmann (2002). The asset can be thought of as a particular product or process. Specifically, the exogenous variable $\theta_{i}$ represents the change in the asset's value that is caused by the venture's success. The variable $\theta_{i}$ is well-known to all players and does not include any monetary cash flow. If $\theta_{i} \geq 0$, then the venture is a complement for the parental company's. If $\theta_{i}<0$, then the venture substitutes the parental company's asset. Note that $\theta_{i}$ may differ among the parental companies. Therefore, a particular venture can be a complement for a parental company, while it is also a substitute for the other. ${ }^{7}$ The venture Chronocam, for instance, that is

\footnotetext{
${ }^{7}$ Chesbrough and Tucci (2002) show that CVC investments may be a complement or a substitute for the internal $\mathrm{R} \& \mathrm{D}$ activities of the parental company. Thus, $\theta_{i}$ can be also interpreted as the impact on the internal $\mathrm{R} \& \mathrm{D}$ activities of the parental company.
} 
financed by Intel's and Bosch's CVCs can be seen as a complement for the first and as a substitute for the latter parental company.

$C V C_{i}$ decides on the nonmonetary support $s_{i} \in(-\infty, \infty)$ at the private cost $c\left(s_{i}\right)=\frac{1}{2} s_{i}^{2}$. Hence, the CVCs have the possibility to nurture (i.e. $s_{i} \geq 0$ ) or sabotage (i.e. $s_{i}<0$ ) the venture, in contrast to Hellmann (2002) and Riyanto and Schwienbacher (2006). ${ }^{8}$ Masulis and Nahata (2009) and Ivanov and Xie (2010) have shown that one reason for sabotage is the obstruction of the survival of a venture that may turn out to be a possible competitor to the parental company. In this model, the nonmonetary support does not have an impact on the success probability $q$. It can be seen as a value added or an increase of the value of the venture, namely $\left(1+s_{i}\right) \cdot\left(R+\theta_{i}\right)$. An investor determines simultaneously with its investment decision the particular support value. The support activities are by and large complex (e.g. mentoring, endorsement to clients) so that they cannot be stated in a contract upon, in contrast to Riyanto and Schwienbacher (2006) and Casamatta and Haritchabalet (2007).

If a contract is offered, then the venture contracts with at least one of the CVCs, which is a simplifying assumption. ${ }^{9}$ However, this assumption is not critical since several empirical studies found evidence that entrepreneurial firms have a high incentive to obtain external funds and nonmonetary support by a CVC. ${ }^{10}$ Only one financing round takes place and all parties have symmetric information.

I characterize subgame-perfect equilibria as the solution concept for the following two investment settings.

\subsection{Stand-Alone Case}

The stand-alone setting is represented by a sequential game with two stages. $C V C_{1}$ decides first between investment and no investment. If $C V C_{1}$ chooses the latter, then $C V C_{2}$ has the opportunity to offer a contract at the next stage. ${ }^{11}$ A stand-alone investment may emerge in markets in which each CVC wants to prevent influence of other corporate investors on the venture. Suppose $C V C_{i}$ finances the venture. The

\footnotetext{
${ }^{8}$ For simplification, I write that the variable $s_{i}$ represents a nonmonetary support although it could be negative.

${ }^{9}$ In contrast to Hellmann (2002), I suppose that the investors have all the bargaining power.

${ }^{10}$ See for example Gompers and Lerner (2000), Maula (2001), Hochberg et al. (2007) and Ivanov and Xie (2010).

${ }^{11}$ The results of the model also hold for a situation in which both investors decide simultaneously on an investment. The only difference is that multiple equilibria occur, which does not enrich the main insights of the model.
} 
expected utility is then given by

$$
U_{i}^{j}=q \cdot\left(R+\theta_{i}\right)\left(1+s_{i}\right)-\frac{1}{2} s_{i}^{2}-1, \text { if } \mathrm{i}=\mathrm{j} .
$$

The superscript $j=1,2$ refer to the particular investor and the subscript $i=1,2$ refers to the utility of the considered CVC. If $C V C_{j}$ finances the venture, then $C V C_{i}$ obtains the externality $\theta_{i}$, without incurring any costs and the risk-free cash flow $r$. $C V C_{i}$ 's expected utility is then given by

$$
U_{i}^{j}=q \theta_{i}\left(1+s_{j}\right)+r-1, \text { if } \mathrm{i} \neq \mathrm{j} .
$$

Note that the change in the asset's value is reinforced by the nonmonetary support of the opponent. For this investment pattern, the innovation component may be described as a positive externality for complements and as a negative externality for substitutes. If both CVCs reject the venture, then each $C V C$ only obtains the risk-free cash flow $U_{i}^{\text {no }}=r-1$.

\subsection{Syndication Case}

In the syndication case, I consider a syndicate, in which both CVCs invest jointly. The CVCs decide simultaneously on the support level $s_{i}$, with $i \in\{1,2\}$. Given this, I show that there is a unique equilibrium in the support levels.

In a second step, I check if this syndicate satisfies the criterion of stability. The syndicate is stable if and only if it represents a Pareto improvement compared to the equilibrium of the stand-alone setting. Hence, the syndicate is acceptable for $C V C_{i}$ if the utility from syndicate is equal or higher than in the stand-alone case. Otherwise, $C V C_{i}$ blocks the syndicate and the stand-alone setting occurs. The expected utility of syndicate member $C V C_{i}$ is given by

$$
U_{i}^{\text {syn }}=q \cdot\left(\frac{1}{2} R+\theta_{i}\right)\left(1+s_{i}+s_{j}\right)+\frac{1}{2} r-\frac{1}{2} s_{i}^{2}-1 .
$$

In a syndicate, $C V C_{i}$ obtains half of the expected cash flow and finances half of the initial outlay. ${ }^{12}$ The rest of the potential fund is invested in the alternative and will yield the expected return $\frac{1}{2} r$. Thus, the primary cost of a syndicate is that both CVCs have to share the generated cash flow of their investment. ${ }^{13}$ The change in

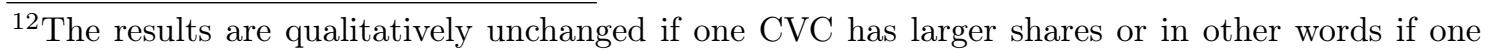
$\mathrm{CVC}$ is the lead investor.

${ }^{13}$ Yung (2012) remarks that the cost of syndication do not only contain the shareholding. Moreover, to put another IVC on inquiry to a investment object may arise a potential competitor. See,
} 
the asset value $\theta_{i}$ is a private value for $C V C_{i}$ and cannot be split. Furthermore, if a syndicate finances the venture, it obtains the support $s_{i}$ of $C V C_{i}$ and additional the support $s_{j}$ of $C V C_{j}$. This assumption is consistent with the value-added hypothesis suggested by Brander et al. (2002). ${ }^{14}$

\section{Equilibrium Analysis}

Without loss of generality, let $\theta_{1} \geq \theta_{2}$. Additionally, I suppose that the venture is a complement for both CVCs $\left(\theta_{i} \in \mathbb{R}_{+}\right)$. I will relax this assumption later and suppose that the venture is a complement for $C V C_{1}\left(\theta_{1} \geq 0\right)$ but a substitute for $C V C_{2}\left(\theta_{2}<0\right)$.

\subsection{Stand-Alone Case}

In the stand-alone case, $C V C_{1}$ decides first whether to invest or not. If $C V C_{1}$ decides on an investment, then the venture obtains funds from this investor and the game ends. On the other hand, if $C V C_{1}$ has not invested, then $C V C_{2}$ can decide on an investment. Consider first the latter case. If $C V C_{2}$ decides to finance the venture, then it provides nonmonetary support in order to increase the value of the venture. I derive the optimal support value $\widehat{s}_{2}$ by solving the respective maximization problem of equation (1):

$$
\widehat{s}_{2}=\underset{s_{2} \in \mathbb{R}}{\operatorname{argmax}}\left\{q \cdot\left(R+\theta_{2}\right)\left(1+s_{2}\right)-\frac{1}{2} s_{2}^{2}-1\right\}
$$

The solution to this problem is given by:

$$
\widehat{s}_{2}=q \cdot\left(R+\theta_{2}\right) .
$$

Note that the weaker (stronger) the complement the lower (higher) is the nonmonetary support of $C V C_{2}$. Likewise, the lower (higher) the expected cash flow the lower (higher) is the nonmonetary support of $C V C_{2}$.

Given that $C V C_{1}$ has not invested, $C V C_{2}$ thus obtains $U_{2}^{2}\left(\widehat{s}_{2}\right)$ if it finances the venture and $U_{2}^{n o}$ otherwise. Comparing these utility levels, the following condition

for instance Casamatta and Haritchabalet (2007) and Cestone et al. (2006).

${ }^{14}$ By contrast, Hellmann (2002) and Casamatta and Haritchabalet (2007) suppose that only the lead investor of a syndicate provides nonmonetary support. 
has to be fulfilled for an investment:

$$
q \cdot\left(R+\theta_{2}\right)\left(1+\widehat{s}_{2}\right)-\frac{1}{2}\left(\widehat{s}_{2}\right)^{2}-1 \geq r-1
$$

Substituting the optimal support $\widehat{s}_{2}$ into expression (5), yields

$$
\begin{gathered}
q \cdot\left(R+\theta_{2}\right)\left(1+q\left(R+\theta_{2}\right)\right)-\frac{1}{2}\left(q\left(R+\theta_{2}\right)\right)^{2}-1 \geq r-1 \\
\theta_{2} \geq \frac{\sqrt{1+2 r}-1}{q}-R \equiv \bar{\theta}_{2} .
\end{gathered}
$$

It is easy to show that $\frac{\partial \bar{\theta}_{2}}{\partial r}>0, \frac{\partial \bar{\theta}_{2}}{\partial q}<0$ and $\frac{\partial \bar{\theta}_{2}}{\partial R}<0$. Provided that $C V C_{1}$ does still not invest at the first stage and the alternative $r$ increases, then only stronger complements obtain funds by $C V C_{2}$ due to an increase in the value of the outside option $U_{2}^{n o}$. On the other hand, if the success probability increases, then weaker complements may be financed by $C V C_{2}$. The same applies for an increase in the expected cash flow. Intuitively, there exists a threshold, for which $C V C_{2}$ decides to finance all complementary ventures, whenever the expected cash flow is above this threshold:

$$
\bar{\theta}_{2}<0 \Leftrightarrow R>\frac{\sqrt{1+2 r}-1}{q}
$$

Working backwards, I investigate the optimal action of $C V C_{1}$. Suppose it decides to invest. Similar to its opponent, $C V C_{1}$ provides nonmonetary support in order to increase the value of the venture. The optimal support is analogously given by $\widehat{s}_{1}=q\left(R+\theta_{1}\right)$.

Suppose now condition (6) is not fulfilled. Then $C V C_{2}$ chooses not to invest in the continuation game in which $C V C_{1}$ does not invest. Thus, $C V C_{1}$ compares the utility levels $U_{1}^{1}\left(\widehat{s}_{1}\right)$ and $U_{1}^{n o}$. The resulting threshold is analogous to condition (6):

$$
\theta_{1} \geq \frac{\sqrt{1+2 r}-1}{q}-R \equiv \bar{\theta}_{1}
$$

Second, I assume that condition (6) is fulfilled. Then $C V C_{2}$ chooses to invest in the continuation game in which $C V C_{1}$ does not invest. Given this, $C V C_{1}$ obtains $U_{1}^{2}\left(\widehat{s}_{2}\right)$. However, if $C V C_{1}$ chooses to invest, then it still obtains $U_{1}^{1}\left(\widehat{s}_{1}\right)$ because it 
decides first. Therefore, $C V C_{1}$ compares $U_{1}^{1}\left(\widehat{s}_{1}\right)$ and $U_{1}^{2}\left(\widehat{s}_{2}\right)$ :

$$
q \cdot\left(R+\theta_{1}\right)\left(1+\widehat{s}_{1}\right)-\frac{1}{2}\left(\widehat{s}_{1}\right)^{2}-1 \geq q \theta_{1}\left(1+\widehat{s}_{2}\right)+r-1
$$

Substituting the optimal supports $\widehat{s}_{1}$ and $\widehat{s}_{2}$ into expression (9), yields

$$
\begin{gathered}
q \cdot\left(R+\theta_{2}\right)\left(1+q\left(R+\theta_{1}\right)\right)-\frac{1}{2}\left(q\left(R+\theta_{1}\right)\right)^{2}-1 \geq q \theta_{1}\left(1+q\left(R+\theta_{2}\right)\right)+r-1 \\
\theta_{2} \leq \frac{R(2+q R)}{2 q \theta_{1}}+\frac{\theta_{1}}{2}-\frac{r}{q^{2} \theta_{1}} \equiv \varphi\left(\theta_{1}\right) .
\end{gathered}
$$

I summarize the above analysis in the following proposition, that states the equilibrium behavior of both CVCs in the stand-alone setting:

Proposition 1 (Stand-alone investment). The stand-alone case has a unique equilibrium, that can be characterized as follows.

(i) No investment occurs if condition (6) and (8) do not hold.

(ii) Suppose condition (6) holds and (7) does not hold. Then $C V C_{2}$ becomes the investor if condition (10) does not hold. Otherwise, $C V C_{1}$ is the investor.

(iii) Suppose condition (7) holds. Then $C V C_{2}$ becomes the investor if condition (10) does not hold. Otherwise, $C V C_{1}$ is the investor.

The equilibrium classes of the stand-alone setting are illustrated in Figure 1: each pair $\left(\theta_{1}, \theta_{2}\right)$ is assigned to the respective investment pattern: stand-alone investment of $C V C_{1}$ and $C V C_{2}$ [Denoted by $I$ ], respectively, or no investment [Denoted by $\chi$ ]. Recall that the venture is a complement for both CVCs and $\theta_{1} \geq \theta_{2}$.

Consider Figure 1 part (b), where condition (7) holds. $C V C_{2}$ finances the venture only if the venture is a strong complement for both investors (i.e. condition (10) is satisfied). In other words, if the expected cash flow is higher, $C V C_{1}$ is better of investing alone for more couples of $\left\{\theta_{1} ; \theta_{2}\right\}$ and not to obtain $\theta_{1}$ plus the alternative $r$ through an investment of the opponent. ${ }^{15}$ The reasoning underlying this result is straightforward: the surplus of an investment is the combination of the expected cash flow and the change in the asset's value, that obtains a premium (value added)

${ }^{15}$ I state for $\varphi\left(\theta_{1}\right)$ the following impact of the other model parameters: $\frac{\partial \varphi\left(\theta_{1}\right)}{\partial r}<0, \frac{\partial \varphi\left(\theta_{1}\right)}{\partial R}>0$ and $\frac{\partial \varphi\left(\theta_{1}\right)}{\partial q}<0$ if $q>\frac{2 r}{R}$ and $\frac{\partial \varphi\left(\theta_{1}\right)}{\partial q}>0$ if $q<\frac{2 r}{R}$. Thus, the success probability has a varied impact on $\varphi\left(\theta_{1}\right)$. The intuition is the following: if $q>\frac{2 r}{R}$ applies, then $\frac{\partial U_{1}^{2}\left(\widehat{s}_{2}\right)}{\partial q}>\frac{\partial U_{1}^{1}\left(\widehat{s}_{2}\right)}{\partial q}$. Therefore, $C V C_{1}$ prefers not to invest for more complements. 


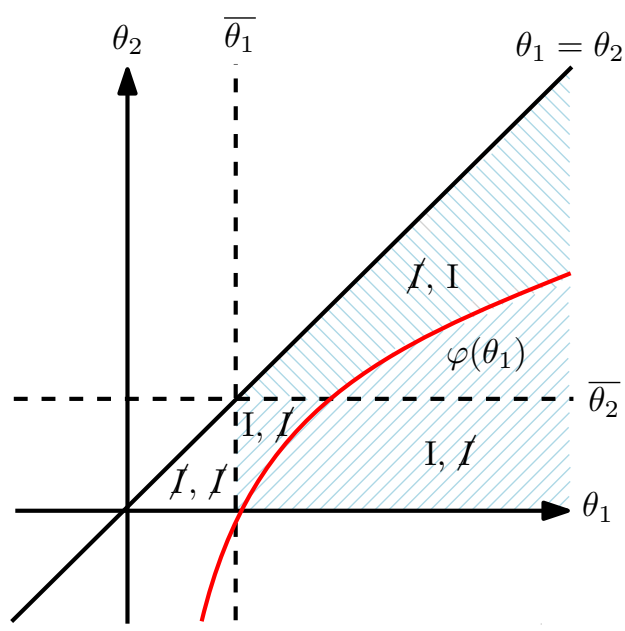

(a)

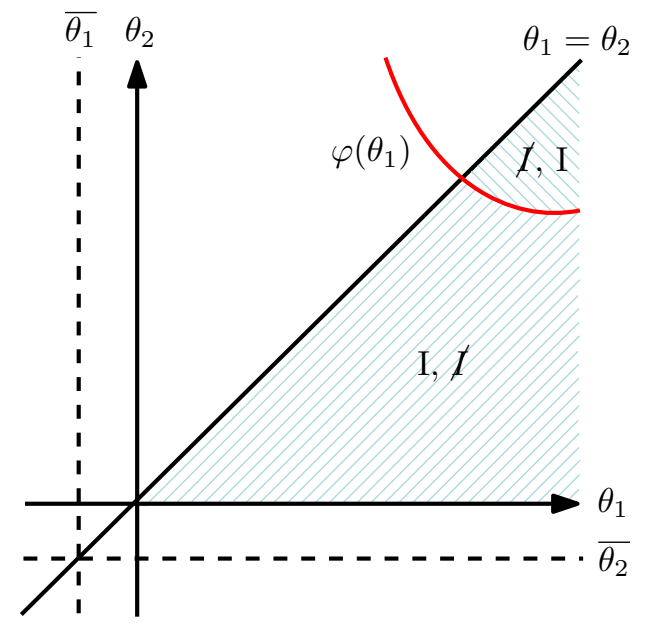

(b)

Figure 1: In Illustration (a), condition (7) does not hold, whereas in (b) condition (7) holds.

through the cost-intensive support of the investor, less the capitalization. If the expected cash flow increases, then the value added increases stronger than the costs of the optimal support. As a consequence, the venture becomes valuable for $C V C_{1}$ for a wider range of the couple $\left\{\theta_{1} ; \theta_{2}\right\}$.

However, the rejection of an investment is without costs. Hence, $\theta_{1}$ may also be viewed as a positive external effect for $C V C_{1}$. In addition, the opponent provides the costly support and increases the value of $\theta_{1}$. This nonmonetary support increases if the expected cash flow and/or the change in the asset's value increase. Therefore, $C V C_{1}$ does not invest if the venture is a strong complement for both investors, i.e. condition (10) is not satisfied, even for a higher expected cash flow.

It seems worth noting that if $C V C_{1}$ invests, then it always provides the same or a higher nonmonetary support than $C V C_{2}$. To see this point, recall that $\theta_{1} \geq \theta_{2}$. Hence, the positive externality may prohibit funding of the investor enabling higher nonmonetary support for the venture. The following corollary of Proposition (1) emphasizes this point.

Corollary 1 If $C V C_{2}$ is the stand-alone investor, then the nonmonetary support is worse-off compared to the support of a stand-alone investor $C V C_{1}$.

\subsection{Syndication Case}

In this section, I consider a syndicate in which each $C V C_{i}$ obtains $U_{i}^{s y n}$. According to the idea of the value-added hypothesis, both CVCs provide nonmonetary support for the venture. The CVCs decide simultaneously on the nonmonetary support level 
$s_{i}$, with $i \in\{1,2\}$. The following proposition shows that there exists a unique equilibrium in support levels in dominant strategies:

Proposition 2 (Syndicate Support). There exists a unique equilibrium in nonmonetary support levels. The equilibrium is given by

$$
s_{i}^{s y n}=q \cdot\left(\frac{1}{2} R+\theta_{i}\right) .
$$

The support $s_{i}^{\text {syn }}$ only depends on the change in the asset's value of $C V C_{i}$. The change in the asset's value of $C V C_{j}$ is not relevant because it is a private value. The weaker (stronger) the own complement the lower (higher) is $s_{i}^{\text {syn }}$. Likewise, the lower (higher) the expected cash flow the lower (higher) is the nonmonetary support of each investor. ${ }^{16}$

I follow Sørensen (2007) by using a stability criterion for the syndicate case. The syndicate is called stable if and only if it represents a Pareto improvement compared to the equilibrium utility of the stand-alone setting. First, suppose the equilibrium of the stand-alone setting is characterized by no investment. Hence, $C V C_{i}$ obtains the stand-alone utility $U_{i}^{n o}$. Then $C V C_{1}$ accepts a syndicate if the following condition is fulfilled:

$$
\begin{gathered}
q\left(\frac{1}{2} R+\theta_{1}\right)\left(1+s_{1}^{\text {syn }}+s_{2}^{\text {syn }}\right)+\frac{1}{2} r-\frac{1}{2}\left(s_{1}^{\text {syn }}\right)^{2}-1 \geq r-1 \\
\theta_{2} \geq \frac{r-1}{q\left(R+2 \theta_{1}\right)}-\frac{\left(3 R+2 \theta_{1}\right)}{4\left(R+2 \theta_{1}\right)} \equiv v_{1}^{*}\left(\theta_{1}\right) .
\end{gathered}
$$

The same approach applies for $C V C_{2}$. This investor accepts a syndicate if the following condition is fulfilled:

$$
\theta_{2} \geq \frac{\sqrt{4 r+\left(2+q\left(R+2 \theta_{1}\right)\right)^{2}}}{2 q}-\frac{1+q R+q \theta_{1}}{q} \equiv v_{2}^{*}\left(\theta_{1}\right)
$$

Second, I turn to the case when $C V C_{1}$ is the stand-alone investor. Hence, $C V C_{1}$ obtains $U_{1}^{1}\left(\widehat{s}_{1}\right)$, whereas $C V C_{2}$ obtains $U_{2}^{1}\left(\widehat{s}_{1}\right)$. There exists no strictly profitable

\footnotetext{
${ }^{16}$ In contrast to Hellmann (2002), the venture has to accept the privately optimal support of the investors, even though it is inefficient and not the first-best solution.
} 
deviation from syndication if the following conditions are fulfilled:

$$
\begin{aligned}
& \theta_{2} \geq \frac{1}{4 q\left(R+2 \theta_{1}\right)}\left(R(4+q R)-\frac{r}{4 q}\right) \equiv v_{1}^{* * *}\left(\theta_{1}\right) \\
& \theta_{2} \geq \frac{\sqrt{4 r-R\left(4 q+q^{2}\left(3 R+4 \theta_{1}\right)\right)}}{2 q} \equiv v_{2}^{* *}\left(\theta_{1}\right)
\end{aligned}
$$

for $C V C_{1}$ and $C V C_{2}$, respectively.

Last, suppose the equilibrium of the stand-alone setting is characterized by a stand-alone investment of $C V C_{2}$. Thus, $C V C_{1}$ obtains $U_{1}^{2}\left(\widehat{s}_{2}\right)$, whereas $C V C_{2}$ obtains $U_{2}^{2}\left(\widehat{s}_{2}\right)$. There exist no strictly profitable deviation from syndication if the following condition are fulfilled:

$$
\begin{aligned}
& \theta_{2} \geq-\frac{1}{q}+\frac{r}{q^{2} R}-\frac{3 R}{4}-\frac{\theta_{1}^{2}}{R} \equiv v_{1}^{* *}\left(\theta_{1}\right) \\
& \theta_{2} \geq \frac{1}{8 q \theta_{1}}\left(R\left(4+q\left(R-4 \theta_{1}\right)\right)-\frac{4 r}{q}\right) \equiv v_{2}^{* * *}\left(\theta_{1}\right)
\end{aligned}
$$

for $C V C_{1}$ and $C V C_{2}$, respectively.

Given the above results, the following lemma establishes useful values of the expected cash flow:

Lemma 1 There exist unique values $\underline{R}, \widetilde{R}, \bar{R} \in \mathbb{R}_{+}$with $\underline{R}<\widetilde{R}<\bar{R}$ such that

- $v_{2}^{*}(0) \leq 0$ if and only if $R \geq \widetilde{R}$,

- $v_{1}^{* *}(0) \leq \bar{\theta}_{2}, v_{2}^{* *}(0) \leq \bar{\theta}_{1}$ and $v_{2}^{*}\left(\overline{\theta_{1}}\right) \leq 0$ if and only if $R \geq \underline{R}$,

- $v_{1}^{* * *}\left(\theta_{1}\right) \leq 0$ if and only if $R \geq \bar{R}$.

Lemma 1 has an immediate consequence for the next result, which shows that the stability of the syndicate depends on the particular expected cash flow value:

Proposition 3 (Syndication for complements).

(i) Suppose the equilibrium of the stand-alone setting is characterized by no investment. Then a syndicate is stable if $R<\widetilde{R}$ and condition (12) holds or if $R \geq \widetilde{R}$

(ii) Suppose the equilibrium of the stand-alone setting is characterized by an investment of $C V C_{1}$. Then a syndicate is stable if $R<\underline{R}$ and condition (14) holds, if $\underline{R} \leq R \leq \bar{R}$ or if $R>\bar{R}$ and condition (13) holds. 
(iii) Suppose the equilibrium of the stand-alone setting is characterized by an investment of $\mathrm{CVC}_{2}$. Then a syndicate is stable if $R<\underline{R}$ and condition (15) holds or if $R \geq \underline{R}$.

Otherwise the syndicate is not stable.

Suppose the equilibrium of the stand-alone setting is characterized by a standalone investment of $C V C_{2}$ and the venture leads to a low expected cash flow (i.e. $R<\underline{R}$ ). Hence, I consider the upper right region of Figure 1 [See, the field with the notation $(\mathbb{X}, \mathrm{I})]$. Condition (15) is the only relevant threshold for the comparison of the syndicate utility with the stand-alone utility. That is, $C V C_{2}$ always accept a syndicate, whereas $C V C_{1}$ considers the threshold $v_{1}^{* *}\left(\theta_{1}\right)$ (i.e. condition (15)). Given this, the latter investor blocks a syndicate for medium complements, i.e. $\theta_{2} \in\left[\bar{\theta}_{2}, v_{1}^{* *}\right)$. This result can be explained as follows: $C V C_{1}$ obtains a value added through the syndicate support, determined by the strength of $C V C_{2}$ 's complement, i.e. a medium value added. On the other hand, $C V C_{1}$ has to bear its share of the investment cost. Intuitively, if the costless impact on the asset plus the alternative $r$ exceeds the surplus of a syndicate, then $C V C_{1}$ blocks the syndicate. Figure 2 illustrates this deviation from syndication. ${ }^{17}$

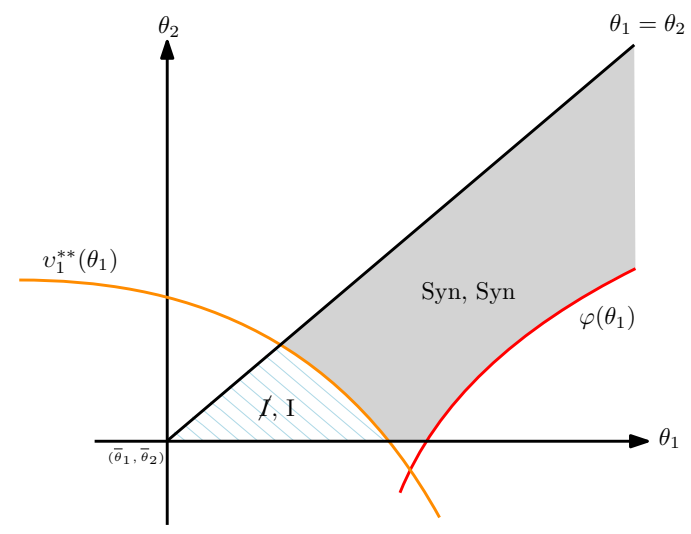

Figure 2: Stability of the syndicate.

In anticipation of the later analysis of higher expected cash flows, I remark that $C V C_{2}$ may be the stand-alone investor only if the expected cash flow is low (i.e. $R<\underline{R}$ ). If the expected cash flow is medium (i.e. $\widetilde{R} \leq R<\bar{R}$ ), then a syndicate is stable for all complements. The surplus of a syndicate is high enough and exceeds the utility from the stand-alone case.

Suppose now that the venture enables a high expected cash flow (i.e. $R \geq \bar{R}$ ). $C V C_{1}$ blocks a syndicate, if $C V C_{2}$ obtains a weak complement, i.e. $\theta_{2} \in\left[0, v_{2}^{* * *}\right)$ due

\footnotetext{
${ }^{17}$ See, the proof of Proposition (3) for a detailed illustration.
} 
to condition (13). The possible nonmonetary support of $C V C_{2}$ is too low to exceed the cost of a syndicate (sharing of the expected cash flow) so that $C V C_{1}$ passes the value added of the opponent. However, for $\theta_{2}$ high enough, i.e. $\theta_{2}>v_{1}^{* * *}\left(\theta_{1}\right)$, a syndicate is stable.

\subsection{Impact of a Substitute}

In the current section I relax the assumption that the venture is a complement for both investors and suppose that $C V C_{2}$ is confronted with a substitute $\left(\theta_{2}<0\right)$. Furthermore, the venture is a complement for $C V C_{1}$. As a preliminary step, I check this modification for the stand-alone case.

\subsubsection{Stand-Alone Case}

$C V C_{2}$ chooses to invest in the continuation game if and only if condition (6) is fulfilled. However, the venture is now a substitute. Hence, the expected cash flow has to be high enough to countervail the negative impact of the substitute on $C V C_{2}$ 's utility (e.g. condition (7) is satisfied). $C V C_{1}$ decides first and compares $U_{1}^{1}\left(\widehat{s}_{1}\right)$ and $U_{1}^{2}\left(\widehat{s}_{2}\right)$. Thus, I check condition (10):

$$
\varphi\left(\theta_{1}\right) \geq 0 \Leftrightarrow R \geq \frac{\sqrt{1+2 r}-1}{q} .
$$

Obviously, if condition (7) is fulfilled, then condition (8) and (10) also hold $\forall \theta_{2}<0$. As a consequence, $C V C_{1}$ decides to finance the venture if $C V C_{2}$ chooses to invest in the continuation game. Second, I suppose that condition (6) is not fulfilled. Then, analogous to Section 2.1, $C V C_{1}$ chooses to invest if condition (8) is fulfilled. Otherwise no investment occurs.

I summarize the above analysis in the following proposition, that states the equilibrium behavior of both CVCs in the stand-alone setting if $C V C_{2}$ is confronted with a substitute:

Proposition 4 (Stand-alone investment for a substitute). No investment occurs if condition (8) does not hold. Otherwise, $C V C_{1}$ is the investor.

The reasoning underlying this result is straightforward. The nonmonetary support of $C V C_{2}$ is reduced by the value $q \cdot \theta_{2}$ in case of an investment in the continuation game. Hence, a stand-alone investment is chosen by $C V C_{1}$ as a best response to an investment of $C V C_{2}$ and its reduced support. 


\subsubsection{Syndication Case}

I proceed with the syndication case. The unique equilibrium in support levels is given by $s_{i}^{s y n}=q \cdot\left(\frac{1}{2} R+\theta_{i}\right)$. Given these support levels there exist a sabotage threshold for $C V C_{2}$ due to the negative impact of the substitute. This threshold is given by

$$
\left|-\theta_{2}\right|>\frac{1}{2} R
$$

Hence, $C V C_{2}$ has now an incentive to hinder the development of the venture if condition (17) is fulfilled. The stronger (weaker) the substitute the stronger (weaker) is the sabotage by $C V C_{2}$.

Previous research on the negative impact of CVCs on their ventures (Masulis and Nahata, 2009; Ivanov and Xie, 2010) provided evidence that CVCs face incentives to obstruct the survival of ventures, that may turn out to be possible competitors. For instance, a parental company uses its CVC to obtain information about the product or services of a venture to develop a competitive asset of its own at the venture's expense. Moreover, Dushnitsky and Shaver (2009) state that corporate investors may oust intellectual property in some cases, originally created by the ventures.

The following proposition entails conditions under which the syndicate setting is stable if $\theta_{1} \geq 0$ and $\theta_{2}<0:^{18}$

Proposition 5 (Syndication for a substitute).

(i) Suppose the equilibrium of the stand-alone setting is characterized by no investment. Then a syndicate is stable if $R \geq \underline{R}$ and condition (12) holds.

(ii) Suppose the equilibrium of the stand-alone setting is characterized by a standalone investment of $C V C_{1}$. Then a syndicate is stable if $R<\underline{R}$ and conditions (13) and (14) hold or if $\underline{R} \leq R<\bar{R}$ and condition (13) holds.

Otherwise the syndicate is not stable.

I now compare the situation where the venture is a complement for both investors with a situation where the venture is complement for $C V C_{1}$ and a substitute for $\mathrm{CVC}_{2}$. Intuitively, a syndicate is less often stable if the possible syndication partner of $C V C_{1}$ is confronted with a substitute due to a lower nonmonetary support. The next proposition emphasizes this point: ${ }^{19}$

\footnotetext{
${ }^{18}$ See, the proof of Proposition (7) for a detailed illustration.

${ }^{19}$ Intuitively, if $\theta_{i}=0$, then the present model is modified to an IVC model. Given this, CVC syndicates lead to a higher value added than IVC syndicates if $\theta_{i}>0$ and $\theta_{j} \geq 0$ or $\theta_{i}>\left|-\theta_{j}\right|$. Otherwise IVC syndicates lead to a higher value added.
} 
Proposition 6 (Corporate venture capital value-added hypothesis). If the venture is a complement for both CVCs, then a syndicate leads to a higher value added than a stand-alone investment. Otherwise a stand-alone investor enables a higher value added.

Brander et al. (2002) formulate their value-added hypothesis in the context of syndicates only consisting of IVCs. I expand their idea to CVC syndicates and take into account the feature of an innovation objective. According to the terminology of Brander et al. (2002), I call this extension the corporate venture capital value-added hypothesis.

For a low up to a medium expected cash flow (i.e. $R<\bar{R}$ ), I find that a syndicate can be stable if $\theta_{1} \geq 0$ and $\theta_{2}<0$. Indeed, if the expected cash flow is high (i.e. $R \geq \bar{R})$, then $C V C_{1}$ does not accept a syndicate. The venture is too attractive in terms of the expected cash flow, such that $C V C_{1}$ blocks a syndicate and finances the venture as a stand-alone investor.

Suppose now a medium expected cash flow occurs (i.e. $\widetilde{R} \leq R<\bar{R}$ ), a syndicate is stable if the venture is a complement for both investors. The nonmonetary support of two CVCs increase the value of the investment, such that both investors accept a syndicate. However, if the venture is a substitute for $C V C_{2}$, the results are less clear. A syndicate is stable for weak substitutes, i.e. $\theta_{2}<\max \left[v_{2}^{*}, v_{1}^{* * *}\right]$, whereby $C V C_{1}$ blocks a syndicate for medium and strong substitutes.

It is important to point out that a syndicate can also be stable if $C V C_{1}$ provides nonmonetary support $\left(s_{1}^{\text {syn }}>0\right)$, whereas $C V C_{2}$ sabotages the development of the venture $\left(s_{2}^{s y n}<0\right)$. This result is stated in the following Proposition.

Proposition 7 (Sabotage by a CVC). Suppose conditions (13), (14) and (17) hold. Then a syndicate is stable if $R<\frac{r}{q \sqrt{1+2 r}}$, such that $C V C_{1}$ provides nonmonetary support, whereas $\mathrm{CVC}_{2}$ sabotages the development of the venture.

To understand this point, recall that the syndicate partner bears a share of the capitalization. This cost saving exceeds the value added of a higher nonmonetary support due to a possible stand-alone investment. As a consequence, $C V C_{1}$ accepts a syndicate and allows $C V C_{2}$ to reduce the negative effect of the venture for itself. The same effect explains why a syndicate is stable for stronger substitutes, if the expected cash flow decreases down to a lower level. 


\subsection{Shareholding Decision}

Consider a modification of the basic model in which the syndicate shares may differ between the CVCs. The shareholding is denoted by $\alpha$ for $C V C_{1}$ and $(1-\alpha)$ for $C V C_{2}$, respectively. I suppose again that the venture is a complement for both CVCs (i.e. $\theta_{i} \in \mathbb{R}_{+}$). Henceforth, the expected utility of a syndicate is written:

$$
\begin{aligned}
& \widetilde{U}_{1}^{s y n}=q \cdot\left(\alpha R+\theta_{1}\right)\left(1+s_{1}+s_{2}\right)-\frac{1}{2} s_{1}^{2}+(1-\alpha) r-1, \\
& \widetilde{U}_{2}^{s y n}=q \cdot\left((1-\alpha) R+\theta_{2}\right)\left(1+s_{1}+s_{2}\right)-\frac{1}{2} s_{2}^{2}+\alpha r-1 .
\end{aligned}
$$

for $C V C_{1}$ and $C V C_{2}$, respectively. Intuitively, the privately optimal support of $C V C_{1}$ is now given by $\widetilde{s}_{1}^{s y n}=q \cdot\left(\alpha R+\theta_{1}\right)$. Analogously, $C V C_{2}$ provides the nonmonetary support $\left.\widetilde{s}_{2}^{s y n}=q \cdot\left((1-\alpha) R+\theta_{2}\right)\right)$. Given the above modification, a syndicate has to fulfil two classes of constraints to satisfy the criterion of stability: a participation constraint (i.e. a syndicate represents a Pareto improvement compared to the equilibrium of the stand-alone setting) and a feasibility constraint (i.e. $\alpha \in$ $[0 ; 1])$.

As a preliminary step, I consider the participation constraint. Suppose that the equilibrium of the stand-alone setting is characterized by no investment. Hence, $C V C_{1}$ obtains the stand-alone utility $U_{1}^{n o} . C V C_{1}$ has no strictly profitable deviation from syndication if the following condition is fulfilled:

$$
q\left(\alpha R+\theta_{1}\right)\left(1+\widetilde{s}_{1}^{s y n}+\widetilde{s}_{2}^{s y n}\right)+(1-\alpha) r-\frac{1}{2}\left(\widetilde{s}_{1}^{s y n}\right)^{2}-1 \geq r-1
$$

Condition (18) is satisfied if $\alpha \in[A-B ; A+B] \equiv \alpha \in\left[\underline{\alpha}_{1}^{n o} ; \bar{\alpha}_{1}^{n o}\right]$, where A and B are given by

$$
\begin{aligned}
& A \equiv \frac{-r+q R+q^{2} R^{2}+q^{2} R \theta_{2}}{q^{2} R^{2}}, \\
& B \equiv \frac{\sqrt{q^{3} R^{2} \theta_{1}\left(2+q\left(2 R+\theta_{1}+2 \theta_{2}\right)\right)+\left(r-q R\left(1+q\left(R+\theta_{2}\right)\right)\right)^{2}}}{q^{2} R^{2}} .
\end{aligned}
$$

Thus, $C V C_{1}$ is sufficiently motivated to join a syndicate and to exert support if it receives a share above the minimum threshold $\max \left\{0, \underline{\alpha}_{1}^{n o}\right\}$. Indeed, the share can be limited to a maximum threshold $\min \left\{1, \bar{\alpha}_{1}^{n o}\right\}$ because of the investment costs in a syndicate. Hence, for particular investment situations, $C V C_{1}$ is better-off to obtain less shares. For $C V C_{2}$, I derive analogously, that the participation constraint 
is satisfied if $\alpha \in[C-D ; C+D] \equiv \alpha \in\left[\underline{\alpha}_{2}^{n o} ; \bar{\alpha}_{2}^{n o}\right]$, where

$$
\begin{aligned}
& C \equiv \frac{r+q r+q^{2} R \theta_{1}}{q^{2} R^{2}}, \\
& D \equiv \frac{\sqrt{q^{3} R^{2} \theta_{1}\left(2+q\left(2 R+\theta_{1}+2 \theta_{2}\right)\right)+\left(r-q R\left(1+q\left(R+\theta_{2}\right)\right)\right)^{2}}}{q^{2} R^{2}}
\end{aligned}
$$

$C V C_{2}$ 's minimum threshold is defined by $\max \left\{0, \underline{\alpha}_{2}^{n o}\right\}$ and the maximum threshold is given by $\min \left\{1, \bar{\alpha}_{2}^{n o}\right\}$.

Second, I turn to the case when $C V C_{1}$ is the stand-alone investor. Hence, $C V C_{1}$ obtains $U_{1}^{1}\left(\widehat{s}_{1}\right)$, whereas $C V C_{2}$ obtains $U_{2}^{1}\left(\widehat{s}_{1}\right)$. For clarity, I skip the particular values of the interval and state that $\alpha \in\left[\max \left\{0, \underline{\alpha}_{1}^{1}\right\}, \min \left\{1, \bar{\alpha}_{2}^{1}\right\}\right]$, such that $C V C_{1}$ has no strictly profitable deviation from syndication. On the other hand, $C V C_{2}$ accepts to syndicate if the following interval is fulfilled: $\alpha \in\left[\max \left\{0, \underline{\alpha}_{2}^{1}\right\}, \min \left\{1, \bar{\alpha}_{2}^{1}\right\}\right]$.

Last, suppose the equilibrium of the stand-alone setting is characterized by a stand-alone investment of $C V C_{2}$. Thus, $C V C_{1}$ obtains $U_{1}^{2}\left(\widehat{s}_{2}\right)$, whereas $C V C_{2}$ obtains $U_{2}^{2}\left(\widehat{s}_{2}\right) . \quad C V C_{1}$ has no strictly profitable deviation from syndication if $\alpha \in\left[\max \left\{0, \underline{\alpha}_{1}^{2}\right\}, \min \left\{1, \bar{\alpha}_{1}^{2}\right\}\right]$. On the other hand, $C V C_{2}$ accepts to syndicate if $\alpha \in\left[\max \left\{0, \underline{\alpha}_{2}^{2}\right\}, \min \left\{1, \bar{\alpha}_{2}^{2}\right\}\right]$

Proposition (8) summarizes the results for the participation constraint under consideration of the feasibility constraint, such that the shareholding is in the interval $\alpha \in[0 ; 1]:$

Proposition 8 (Syndication for complements with variable shares). There exist unique values $\widehat{R}^{n o}, R_{i}^{n o}, R_{i}^{1}$ and $R_{i}^{2} \in \mathbb{R}_{+}$with $\widehat{R}^{n o}<R_{1}^{n o}<R_{2}^{n o}, R_{1}^{1}>R_{1}^{2}$ and $R_{2}^{1}<R_{2}^{2}$ such that the following statements hold:

(i) Suppose the equilibrium of the stand-alone setting is characterized by no investment. Then a syndicate is stable

- for $\alpha \in[0 ; 1]$ if and only if $R>R_{2}^{n o}$,

- for $\alpha \in\left[\underline{\alpha}_{2}^{n o} ; 1\right]$ if and only if $R_{1}^{n o}<R \leq R_{2}^{n o}$,

- for $\alpha \in\left[\underline{\alpha}_{2}^{n o} ; \bar{\alpha}_{1}^{n o}\right]$ if and only if $\widehat{R}^{n o}<R \leq R_{1}^{n o}$.

(ii) Suppose the equilibrium of the stand-alone setting is characterized by an investment of $C V C_{1}$. Then a syndicate is stable

- for $\alpha \in[0 ; 1]$ if and only if $R_{2}^{1}<R \leq R_{1}^{1}$,

- for $\alpha \in\left[\underline{\alpha}_{1}^{1} ; 1\right]$ if and only if $R>R_{1}^{1}$, 
- for $\alpha \in\left[\underline{\alpha}_{2}^{1} ; 1\right]$ if and only if $R \leq R_{2}^{1}$.

(iii) Suppose the equilibrium of the stand-alone setting is characterized by an investment of $C V C_{2}$. Then a syndicate is stable

- for $\alpha \in[0 ; 1]$ if and only if $R_{1}^{2}<R \leq R_{2}^{2}$,

- for $\alpha \in\left[0 ; \bar{\alpha}_{2}^{2}\right]$ if and only if $R>R_{2}^{2}$,

- for $\alpha \in\left[0 ; \bar{\alpha}_{1}^{2}\right]$ if and only if $R \leq R_{1}^{2}$.

Otherwise the syndicate is not stable.

To understand the intuition, suppose the equilibrium of the stand-alone setting is characterized by no investment. The results show that a syndicate is stable for all feasible shares (i.e. $\alpha \in[0 ; 1]$ ) if the the expected is high enough (i.e. $R>R_{2}^{n o}$ ). However, if the expected cash flow decreases, then the interval for stable syndicates becomes limited by both particular shareholding benchmarks (i.e. $\underline{\alpha}_{2}^{n o}$ and $\bar{\alpha}_{1}^{n o}$ ). Indeed, if the expected cash flow decreases strongly (i.e. $R<R^{n o}$ ) then the syndicate is blocked for all feasible shares, because the syndication costs are too high. Hence, both investors are better of not to invest.

Suppose now that at least one of the CVCs finance the venture. Intuitively, the particular investor blocks a syndicate if the expected cash flow is high enough and the assigned shareholding is only low (i.e. $\alpha<\underline{\alpha}_{1}^{1}$ for investor $C V C_{1}$ and $\alpha>\underline{\alpha}_{2}^{2}$ for investor $C V C_{2}$ ). It is important to point out that a syndicate is also possible if the expected cash flow decreases strongly. Stand-alone investor $C V C_{1}$, for instance, may syndicate with $C V C_{2}$ if $R<R_{2}^{1}$. However, the latter investor blocks a high shareholding for itself (i.e. $\alpha<\alpha_{2}^{1}$ ) due to the low expected cash flow.

\subsubsection{Pareto Efficient Shareholding Allocation}

I now check which shareholding agreements represents the pareto efficient allocation, in the sense that it is impossible to reallocate the shareholding so as to make one CVC better-off without making the other CVC worse-off. As a preliminary step, I state the following derivations:

$$
\begin{aligned}
& \frac{\partial \widetilde{U}_{1}^{\text {syn }}}{\partial \alpha}=\frac{-r+q R\left(1+q R\left(1+q\left(R+\theta_{2}\right)\right.\right.}{q^{2} R^{2}} \equiv \alpha_{1}^{p e}, \\
& \frac{\partial \widetilde{U}_{2}^{\text {syn }}}{\partial \alpha}=\frac{r-q R\left(1+q \theta_{1}\right)}{q^{2} R^{2}} \equiv \alpha_{2}^{p e} .
\end{aligned}
$$


for $C V C_{1}$ and $C V C_{2}$, respectively. Given this, I consider $C V C_{1}$ and show the following conditions $\forall \alpha \in[0 ; 1]$ :

$$
\begin{aligned}
\frac{\partial \widetilde{U}_{1}^{\text {syn }}}{\partial \alpha}>0 \Leftrightarrow R>\frac{r}{q\left(1+q \theta_{2}\right)}, \\
\frac{\partial \widetilde{U}_{1}^{\text {syn }}}{\partial \alpha}<0 \Leftrightarrow R<\frac{-q-q^{2} \theta_{2}+q \sqrt{1+4 r+2 q \theta_{2}+q^{2} \theta 2^{2}}}{2 q^{2}} .
\end{aligned}
$$

Hence, if condition (19) is fulfilled, then $C V C_{1}$ is better-off to obtain more shares of the syndicate due to the high expected cash-flow. On the other hand, if condition (20) holds, then $C V C_{1}$ is better-off to obtain less shares. However, if both conditions are not fulfilled, then an increase of the shareholding has only a positive impact on the expected cash flow for a particular range of the feasible shares. The threshold for this range is $\alpha_{1}^{p e}$ and $\alpha_{2}^{p e}$, respectively. Second, I turn to $C V C_{2}$ and show the following conditions $\forall \alpha \in[0 ; 1]$ :

$$
\begin{aligned}
& \frac{\partial \widetilde{U}_{2}^{s y n}}{\partial \alpha}>0 \Leftrightarrow R<\frac{-q-q^{2} \theta_{1}+q \sqrt{1+4 r+2 q \theta_{1}+q^{2} \theta_{1}^{2}}}{2 q^{2}}, \\
& \frac{\partial \widetilde{U}_{2}^{s y n}}{\partial \alpha}<0 \Leftrightarrow R>\frac{r}{q\left(1+q \theta_{1}\right)} .
\end{aligned}
$$

The intuition of these conditions are the same as for $C V C_{1}$. Note that condition (22) is always fulfilled if (19) holds due to $\theta_{1} \geq \theta_{2}$. Analogously, condition (21) is always fulfilled if (20) holds. Moreover, condition (21) cannot hold if (22) holds and condition (20) cannot hold if (19) holds, respectively. I now compare condition (19) and (21):

$$
\begin{array}{r}
\frac{r}{q\left(1+q \theta_{2}\right)} \geq \frac{-q-q^{2} \theta_{1}+q \sqrt{1+4 r+2 q \theta_{1}+q^{2} \theta_{1}^{2}}}{2 q^{2}} \\
\theta_{1} \leq \frac{-1-2 r-q \theta_{2}+\sqrt{4 r+\left(1+q \theta_{2}\right)^{2}}}{q+q^{2} \theta_{2}-q \sqrt{4 r+1\left(1+q \theta_{2}\right)^{2}}}
\end{array}
$$

Given this, there exist two different cases for the order of the above thresholds. I proceed with Proposition (9) and summarize the results:

Proposition 9 (Pareto efficient shareholding allocation). I consider a stable syndicate.

(i) Suppose condition (23) is fulfilled.

- If condition (19) does not hold and (22) holds, then every shareholding $\alpha \in\left[0 ; \alpha_{1}^{p e}\right]$ is pareto efficient. 
- If condition (20) and (22) do not hold, then every shareholding $\alpha \in$ $\left[\min \left\{\alpha_{1}^{p e}, \alpha_{2}^{p e}\right\} ; \max \left\{\alpha_{1}^{p e}, \alpha_{2}^{p e}\right\}\right]$ is pareto efficient.

- If condition (20) holds, whereas (20) does not, then every shareholding $\alpha \in\left[\alpha_{2}^{p e} ; 1\right]$ is pareto efficient.

(iii) Suppose condition (23) is not fulfilled.

- If condition (19) and (20) do not hold, then every shareholding $\alpha \in\left[0 ; \alpha_{1}^{p e}\right]$ is pareto efficient.

- If condition (21) and (22) do not hold, then every shareholding $\alpha \in\left[\alpha_{2}^{p e} ; 1\right]$ is pareto efficient.

Otherwise every shareholding $\alpha \in[0 ; 1]$ is pareto efficient.

To understand the intuition of Proposition (9), suppose condition (19) holds, then the CVCs may obtain a high expected cash flow in a stable syndicate. Hence, every shareholding $\alpha \in[0 ; 1]$ is pareto efficient because both investors prefer to obtain more shares. Or in other words, it is impossible to reallocate the shareholding so as to make one CVC better-off without making the other CVC worse-off. On the other hand, if condition (21) holds, then the CVCs may obtain a low expected cash flow. The CVCs prefer to obtain less shares due to the investment costs. Thus, every shareholding $\alpha \in[0 ; 1]$ is pareto efficient.

I turn next to the case, where the change in the asset's value of $C V C_{1}$ is small (e.g. condition (23) holds). If the expected cash flow becomes lower (e.g. condition (19) and (20) do not hold) and $\alpha \in\left[\alpha_{1}^{p e} ; 1\right]$, then the shareholding of $C V C_{1}$ can be reallocated so as to make $C V C_{1}$ better-off. More precisely, $C V C_{1}$ is better-off not to obtain a very high shareholding. On the other hand, $C V C_{2}$ is better off to obtain more shares because condition (22) holds. Hence, the reallocation of the shareholding also makes $C V C_{2}$ better-off. Given this, the range of pareto efficient shares is limited to the $\widetilde{\mathrm{A}} \cap$ nterval $\alpha \in\left[0 ; \alpha_{1}^{p e}\right]$.

\subsubsection{Joint Utility Maximization}

I now check which shareholding allocation maximizes the joint utility of a stable syndicate. It takes into account the participation and feasibility constraint of the CVCs. ${ }^{20}$ First, I consider the joint utility of a syndicate, that should be divided

\footnotetext{
${ }^{20}$ Note that this shareholding can be denoted by second best shareholding due to the participation and feasibility constraint of the CVCs.
} 
between the CVCs:

$$
U^{j o i n t}=q \cdot\left(R+\theta_{1}+\theta_{2}\right)\left(1+\widetilde{s}_{1}^{s y n}+\widetilde{s}_{2}^{s y n}\right)-\frac{1}{2}\left(\widetilde{s}_{1}^{\text {syn }}\right)^{2}-\frac{1}{2}\left(\widetilde{s}_{2}^{s y n}\right)^{2}+r-2 .
$$

Given this, I show the shareholding allocation among the investors that maximize the joint utility:

$\alpha^{\max }=\underset{\alpha \in[0 ; 1]}{\operatorname{argmax}}\left\{q \cdot\left(R+\theta_{1}+\theta_{2}\right)\left(1+\widetilde{s}_{1}^{\text {syn }}+\widetilde{s}_{2}^{\text {syn }}\right)-\frac{1}{2}\left(\widetilde{s}_{1}^{\text {syn }}\right)^{2}-\frac{1}{2}\left(\widetilde{s}_{2}^{\text {syn }}\right)^{2}+r-2\right\}$,

where

$$
\begin{aligned}
& \widetilde{s}_{1}^{\text {syn }}=q \cdot\left(\alpha R+\theta_{1}\right), \\
& \left.\widetilde{s}_{2}^{\text {syn }}=q \cdot\left((1-\alpha) R+\theta_{1}\right)\right) .
\end{aligned}
$$

The solution to this problem is given by:

$$
\alpha^{\max }=\frac{1}{2}+\frac{\theta_{2}-\theta_{1}}{2 R} .
$$

It is important to point out that the shareholding $\alpha^{\max }$ balances the change in the asset's value. In other words, if the innovation advantage of $C V C_{1}$ increases, then it obtains a lower share on the expected cash flow. The same applies if the innovation advantage of $\mathrm{CVC}_{2}$ decreases. However, an increase in the expected cash flow reduces the impact of the difference in the innovation advantage on $\alpha^{\max }$. Hence, if the expected cash flow becomes very high, then it is efficient to split the shareholding equally. Intuitively, if the innovation advantage has the same value for both CVCs (i.e. $\theta_{1}=\theta_{2}$ ), then each investor also obtains half of the shares. On the other hand, if one CVC has a lower innovation advantage in comparison with the syndication partner, then the former investor obtains more shares. Hence, the occurrence of a lead investor (i.e. $\alpha<\frac{1}{2}$ ) in a syndicate with several CVCs may maximize the joint utility, given that the innovation advantage differs among the investors.

Note that the share $\alpha^{\max }$ has an upper bound with $\alpha=\frac{1}{2}$ that occurs if $\theta_{1}=\theta_{2}$ or if the expected cash flow is very high. For other parameters combinations, $C V C_{2}$ 's becomes the lead investor because I suppose that $\theta_{1} \geq \theta_{2}$. However, there exists a 
threshold, such that $\alpha^{\max } \geq 0$ :

$$
R \geq \theta_{1}-\theta_{2}
$$

The following proposition entails conditions under which the shareholding $\alpha^{\max }$ can be achieved given that the syndicate setting satisfies the criterion of stability:

Proposition 10 (Second-best shareholding allocation). Suppose condition (25) holds. The shareholding $\alpha^{\max }$ can be established for a stable syndicate

(i) if the equilibrium of the stand-alone setting is characterized by no investment and $R>R_{2}^{\text {no }}$.

(ii) if the equilibrium of the stand-alone setting is characterized by an investment of $C V C_{1}$ and $R_{2}^{1} \leq R \leq R_{1}^{1}$.

(iii) if the equilibrium of the stand-alone setting is characterized by an investment of $C V C_{2}$ and $R_{1}^{2} \leq R \leq R_{2}^{2}$.

The results show that the shareholding, that maximize the joint utility of a stable syndicate, can be established for all possible equilibria of the stand-alone setting. Indeed, the expected cash flow has to be suitable such that both investors accept a joint investment.

\subsubsection{Nash Bargaining Solution}

Finally, I endogenize the shareholding decision of the syndicate partners. Following Casamatta and Haritchabalet (2007), I use the Nash bargaining solution as negotiation concept for the shareholding allocation. ${ }^{21}$

The Nash bargaining solution is an approach for a two-person bargaining problem. In the present model, the CVCs divide the shares of a syndicate to participate in the syndicate surplus. If negotiation succeeds, then the CVCs conclude a contract on the shareholding. Note that the shareholding allocation is restricted, such that the CVCs are better-off compared to the disagreement point. This disagreement point is the equilibrium of the stand-alone setting or in other words the utility the CVCs can expect to receive if the negotiation breaks down.

Suppose the disagreement point is characterized by no investment, then the Nash bargaining solution can be determined for $C V C_{1}$ through the following maximization

$\overline{{ }^{21} \text { See, for instance Laengle and Loyola }}$ (2012) for a bargaining problem with externalities. 
problem:

$$
\widehat{\delta}=\underset{\delta \in[0 ; 1]}{\operatorname{argmax}}\left\{\Delta U_{1}^{n o} \cdot \Delta U_{2}^{n o}\right\} \Leftrightarrow \widehat{\delta}=\frac{1}{2}
$$

where

$$
\begin{aligned}
& \Delta U_{1}^{n o}=\delta U^{j o i n t}-U_{1}^{n o} \\
& \Delta U_{2}^{n o}=(1-\delta) U^{j o i n t}-U_{2}^{n o} .
\end{aligned}
$$

The parameter $\delta$ is $C V C_{1}$ 's and $(1-\delta) C V C_{2}$ 's share in the joint profit, respectively. Intuitively, both investors equally divide the joint profits due to the same disagreement point. However, the syndication contract only includes a shareholding on the expected cash flow. I have to determine the particular share $\alpha$ for $C V C_{1}$ :

$$
\widetilde{U}_{1}^{\text {syn }}=\frac{1}{2} U^{\text {joint }} \Leftrightarrow \alpha=\frac{-2 r+g\left(R-\theta_{1}+\theta_{2}\right)\left(2+q 8 R+\theta_{1}+\theta_{2}\right)}{2\left(-2 r+q R\left(2+q\left(R+\theta_{1}+\theta_{2}\right)\right)\right)}
$$

I proceed with the case that the disagreement point is characterized by an investment of $C V C_{1}$. Then the following utility differences exist: $\Delta U_{1}^{1}=\delta U^{j o i n t}-U_{1}^{1}$ and $\Delta U_{2}^{1}=(1-\delta) U^{j o i n t}-U_{2}^{1}$, respectively. For clarity, I only state the solution for the shareholding on the expected cash flow:

$$
\alpha=\frac{-4 r+q\left(2 q R^{2}+2 R\left(2+q \theta_{1}\right)+q \theta_{2}\left(\theta_{2}-2 \theta_{1}\right)\right)}{2\left(-2 r+q R\left(2+q\left(R+\theta_{1}+\theta_{2}\right)\right)\right)}
$$

Last, suppose the disagreement point is characterized by an investment of $C V C_{2}$. The same approach holds as for the previous step. Thus, I only show the particular shareholding:

$$
\alpha=\frac{-q^{2} \theta_{1}\left(-2 R+\theta_{1}-2 \theta_{2}\right)}{2\left(-2 r+q R\left(2+q\left(R+\theta_{1}+\theta_{2}\right)\right)\right)}
$$

Given this, I want to justify the share $\alpha^{\max }$ that maximizes the joint surplus of a syndicate by means of bargaining. Hence, I use the analysis for the feasible set of the shareholding (i.e. Proposition (8)) and the conditions under which the shareholding $\alpha^{\max }$ can be established for the feasible set (i.e. Proposition (10)). In this way, Proposition (11) entails conditions under which the shareholding $\alpha^{\max }$ can be implemented by the Nash bargaining solution for a stable syndicate:

Proposition 11 (Nash bargaining solution). There exist unique values $\widetilde{\theta_{1}}$ and $\check{\theta_{1}}$, such that the following statements hold: 
(i) Suppose the equilibrium of the stand-alone setting is characterized by no investment. Then the Nash bargaining solution implements the shareholding $\alpha^{\text {max }}$ for a stable syndicate if and only if equation (26) is fulfilled, $R>R_{2}^{\text {no }}$ and $\theta_{1}=\theta_{2}$.

(ii) Suppose the equilibrium of the stand-alone setting is characterized by an investment of $C V C_{1}$. Then the Nash bargaining solution implements the efficient shareholding for a stable syndicate if and only if equation (27) and condition (25) are fulfilled, $R_{2}^{1} \leq R \leq R_{1}^{1}$ and $\theta_{1}=\widetilde{\theta_{1}}$.

(iii) Suppose the equilibrium of the stand-alone setting is characterized by an investment of $\mathrm{CVC}_{2}$. Then the Nash bargaining solution implements the efficient shareholding for a stable syndicate if and only if equation (28) and condition (25) are fulfilled, $R_{1}^{2} \leq R \leq R_{2}^{2}$ and $\theta_{1}=\check{\theta}_{1}$.

It seems worth noting that there exists a positive external effect for the rejecting investor in case of the last two points of Proposition (11), due to the costless change in the asset's value. Hence, the possibility to bargain for the shares may be viewed as an internalization of this externality. Henceforth, after bargaining, the formerly rejecting investor joins a syndicate and bears some of the investment costs.

However, Proposition (11) states that the Nash bargaining solution only achieve the shareholding $\alpha^{\max }$ in a few special cases and fails in general, respectively. Clearly, transfer payments between the CVCs may be a possibility to increase the number of solutions. $^{22}$ Nevertheless, I view syndicate agreements with transfers as less likely for venture capital investments, following Casamatta and Haritchabalet (2007).

\section{Numerical Examples}

To study the endogenous shareholding issue, consider the following examples of a possible syndication contract. Suppose the disagreement point is characterized by an investment of $C V C_{1}$. For both examples apply $R=5, r=4, q=0.4$ and different couples of $\left\{\theta_{1}, \theta_{2}\right\} .^{23}$

\begin{tabular}{|c|c|c|c|c|}
\hline & $\left\{\theta_{1}, \theta_{2}\right\}$ & Feasible Shares & Pareto Efficient Shares & $\alpha^{\max }$ \\
\hline (i) & $\{2.7,2\}$ & $\alpha \in[0 ; 1]$ & $\alpha \in[0 ; 0.9]$ & 0.33 \\
\hline (ii) & $\{0.55,0.5\}$ & $\alpha \in[0 ; 1]$ & $\alpha \in[0.39 ; 0.6]$ & 0.49 \\
\hline
\end{tabular}

\footnotetext{
${ }^{22}$ See, for instance, Bayar and Chemmanur (2011) for the use of transfer payments in the context of venture capital.

${ }^{23}$ I only focus on the shareholding and do not check if the expected cash flow of the stand-alone case is lower than the the expected cash flow of the syndicate case. Intuitively, the latter case has a higher expected cash flow due to the Nash bargaining approach.
} 
In Example (i), both investors obtain a stronger change in the assets values than in Example (ii). However, $C V C_{1}$ obtains a stronger increase in $\theta_{1}$ than $C V C_{2}$ in $\theta_{2}$. Hence, the former investor obtains less shares in a syndicate that maximizes the joint surplus of a syndicate due to equation (24). On the other hand, in Example (ii), $C V C_{1}$ obtains more shares than in Example (i) because the change in the assets values is almost similar. Thus, these examples illustrate the balancing effect of the shareholding, in the sense that the change in the asset's value becomes balanced by the syndicate shares.

\section{Empirical Predictions}

The findings of the present model allows us to derive some empirical predictions about the investment decision of CVCs that are confronted with innovation objectives:

- First, a central prediction of the model is that the possible innovation objectives have a great influence on the investment decision of CVCs. Sharifzadeh and Walz (2012) provide in their empirical evidence that only a small number of CVCs syndicate with other CVCs. Most of the corporate investments are stand-alone investments. Dushnitsky (2008) gives an example for syndicates, consisting of different corporate investors: the Linux company Red Hat is financed by Compaq, IBM, Intel, Novell, Oracle and SAP. Moreover, MacMillan et al. (2012) remark that only $30 \%$ of their sample classify other CVCs as important syndication partners. However, both studies analyze the CVCs' investment behavior without consideration of the innovation objectives. By contrast, Masulis and Nahata (2009) take this objectives into account and stress that CVCs finance by the majority weak complements. Interestingly, their sample demonstrates that substitutes also obtain in a considerable size investments by CVCs. Unfortunately, Masulis and Nahata (2009) do not differ between different investment pattern (i.e. syndication and stand-alone investment).

Thus, an original feature of the present model is the combination of the investment decision of CVCs with their innovation objectives. Moreover, I investigate the impact of the expected cash flow. I predict that CVCs faced with weak complements but high expected cash flows prefer stand-alone investments. The above empirical evidence is consistent with this result. By contrast, the model predicts that ventures leading to a medium expected cash 
flow are financed by a syndicate without any consideration of the innovation objective. If a substitute is included in the analysis, then stand-alone investments becomes more probable because of the negative impact of the substitute on the nonmonetary support of the affected investor. Nevertheless, syndicates are possible if the expected cash flow is on a low or medium level. This result holds even if the provided nonmonetary support is lower than in the standalone case.

- Brander et al. (2002) show for IVCs that syndicates have significantly higher cash flows than stand-alone investments because of the nonmonetary support (see, the value-added hypothesis) by different investors. The empirical predictions of the present CVC model are less clear. If the venture is a complement for both investors, then a syndicate leads to a higher cash flow because both investors have an incentive to nurture their new investment. By contrast, if one CVC is confronted with a substitute, the opposite result is possible. The investor with the substitute has an incentive to sabotage the venture and to protect the parental company. Thus, a lower cash flow exists. Masulis and Nahata (2009) stress the sabotage motivation of CVCs, but it is still an empirical issue that syndicates with substitutes lead to lower cash flows than stand-alone investments of complementary investors. I call this prediction the corporate venture capital value-added hypothesis.

- Hellmann (2002) formulates an extension of his basic model with two corporate investors and one IVC. The model shows that if the venture is a complement to both large companies, only the CVC with the stronger complement invests. Some examples of CVC investments show that other results than those by Hellmann are possible. One example is the young FinTech firm Gini, that is supported by only one corporate investor, namely, the Main Incubator founded by the Commerzbank. After Gini has succeeded with its banking software for giro accounts, the online bank ING DiBa has established Gini's software before the Commerzbank uses this product for itself (cf. Ing DiBa 2016, Main Incubator 2016). ${ }^{24}$ Obviously, Gini's product is a stronger complement to online banks than to the Commerzbank, a retail bank. However, only Commerzbank finances the young firm. The present model explains this puzzling behavior of the ING DiBa due to the costless impact on some assets of the incumbent company if another CVC bear the investment costs. However, I predict that

\footnotetext{
${ }^{24}$ The bank ING DiBa does not own a subsidiary for corporate venture capital investments. However, investments in young FinTechs are realized by the incumbent company itself.
} 
such an investment behavior only occurs if the venture has a low expected cash flow.

\section{Conclusion}

I examine the investment decision of two CVCs that are subsidiaries of incumbent companies. The CVCs have to decide whether to finance a wealthless venture or not. Two different investment pattern may occur: a stand-alone investment or a syndicate. In both cases, the CVCs provide nonmonetary support to increase the value of the venture. Hence, the advantages of a syndicate entail cost sharing and also the nonmonetary support of the partner. However, both CVCs has to take into account that they share the expected cash flow if they syndicate.

The success of the venture affects some of the parental companies' assets (e.g. a product or process). This change in the asset's value may be positive or negative and differ among the parental companies. Thus, the venture can be a weak complement for a parental company, while it is also a strong complement for the other. I find that a CVC may forgo to finance a complementary venture even if the complementarity is stronger than it is for the other CVC. To understand this point, see that the impact on the assets emerges costless if the venture is financed by the opponent and it succeeds. Given this, the change in the asset's value may be interpreted as a positive external effect due to the absence of a cost contribution. Moreover, if the venture generates a medium expected cash flow, then it is always financed by a syndicate independently of the change in the asset's value. However, this situation only arises if the venture is a complement for both parental companies due to the beneficial support-effect of several investors.

Indeed, corporate investors do not always have a positive impact on their ventures. Syndicates may also established by CVCs with countervailing incentives because of the change in the asset's value. Therefore, if one CVC obtains a positive change, whereas the other obtains a negative change, then a stand-alone investments of the former CVC leads to a higher nonmonetary support. However, in some cases cost sharing eclipse this value added, so that a syndicate occurs.

Finally, the model shows that the division of the shares in a syndicate can balance the change in the asset's value among the CVCs. Hence, if this change occurs as a positive external effect, the allocation of the shareholding may internalizes this externality. 


\section{Appendix A. Proofs}

Proof of Proposition 2.

I consider a syndicate, in which each $C V C_{i}$ obtains

$$
U_{i}^{\text {syn }}=q \cdot\left(\frac{1}{2} R+\theta_{i}\right)\left(1+s_{i}+s_{j}\right)+\frac{1}{2} r-\frac{1}{2} s_{i}^{2}-1 .
$$

The optimal support value $s_{i}^{\text {syn }}$ is given by:

$$
s_{i}^{\text {syn }}=\underset{s_{i} \in \mathbb{R}}{\operatorname{argmax}}\left\{q \cdot\left(\frac{1}{2} R+\theta_{i}\right)\left(1+s_{i}+s_{j}\right)+\frac{1}{2} r-\frac{1}{2} s_{i}^{2}-1\right\} .
$$

For syndicate member $C V C_{i}$ this yields:

$$
s_{i}^{s y n}=q \cdot\left(\frac{1}{2} R+\theta_{i}\right)
$$

This support level dominates all other support levels, regardless of what $C V C_{j}$ does, and is hence a best response to the support $s_{j}^{\text {syn }}$. There is thus a unique equilibrium in nonmonetary support levels.

Proof of Lemma 1. To proof Lemma 1, I characterize different thresholds for the expected cash flow:

- Start with the case where $v_{2}^{*}(0) \leq 0$ :

$$
v_{2}^{*}(0) \leq 0 \Leftrightarrow R \geq \frac{2}{3}\left(\frac{\sqrt{1+3 r}}{q}-\frac{1}{q}\right) \equiv \widetilde{R} .
$$

- I proceed with the case where $v_{1}^{* *}(0) \leq \bar{\theta}_{2}$ and $v_{2}^{* *}(0) \leq \bar{\theta}_{1}$ :

$$
v_{2}^{* *}(0) \leq \overline{\theta_{1}} \Leftrightarrow R \geq \frac{2(\sqrt{1+2 r}-\sqrt{(1+r)})}{q} \equiv \underline{R} .
$$

Equivalently, $v_{1}^{* *}(0) \leq \bar{\theta}_{1}$ and $v_{2}^{*}\left(\overline{\theta_{1}}\right) \leq 0$ if and only if $R \geq \underline{R}$.

- Last, I check the case where $v_{1}^{* * *}\left(\theta_{1}\right) \leq 0$

$$
v_{1}^{* * *}\left(\theta_{1}\right) \leq 0 \Leftrightarrow R \geq \frac{2(\sqrt{1+r}-1)}{q} \equiv \bar{R}
$$


Last, I check the order of the different thresholds for the expected cash flow:

$$
\begin{aligned}
& \widetilde{R} \geq \underline{R} \Leftrightarrow r \geq 0 . \\
& \bar{R} \geq \widetilde{R} \Leftrightarrow r \geq 0 . \\
& \bar{R} \geq \underline{R} \Leftrightarrow r \geq 0 .
\end{aligned}
$$

Hence, the following order exists: $\underline{R}<\widetilde{R}<\bar{R}$.

Proof of Proposition 3.

(i) Suppose that conditions (6) and (8) do not hold, i.e. the equilibrium of the stand-alone setting is characterized by no investment. One checks easily that $v_{2}^{*}\left(\theta_{1}\right)>v_{1}^{*}\left(\theta_{1}\right) \forall \theta_{1} \geq \theta_{2}$. In other words, if $C V C_{2}$ accepts a syndicate, then the same applies for $C V C_{1}$. Also, $0<v_{2}^{*}\left(\theta_{1}\right)<\bar{\theta}_{2}$ since $R<\widetilde{R}$. Therefore, a syndicate is stable

- if $R<\widetilde{R}$ and condition (12) holds,

- if $R \geq \widetilde{R}$.

Otherwise the syndicate is not stable.

(ii) Suppose that condition (6) does not hold and (8) holds or that condition (6) and (10) hold, i.e. the equilibrium of the stand-alone setting is characterized by an investment of $C V C_{1}$. Hence, I consider $v_{1}^{* * *}\left(\theta_{1}\right)$ and $v_{2}^{* *}\left(\theta_{1}\right)$. One checks easily that $\varphi\left(\theta_{1}\right)>v_{1}^{* * *}\left(\theta_{1}\right) \forall \theta_{1} \geq 0$. Therefore, a syndicate is stable

- if $R<\underline{R}$ and condition (14) holds. I have not to consider (13) due to $R<\bar{R}$.

- if $\underline{R} \leq R \leq \bar{R}$.

- if $R>\bar{R}$ and condition (13) holds. I have not to consider (14) due to $\underline{R}<\bar{R}$.

Otherwise the syndicate is not stable.

(iii) Suppose that condition (6) holds and (10) does not hold, i.e. the equilibrium of the stand-alone setting is characterized by an investment of $C V C_{2}$. One checks easily that $\varphi\left(\theta_{1}\right)>v_{2}^{* * *}\left(\theta_{1}\right) \forall \theta_{1} \geq 0$.

Note that I assume $\theta_{1} \geq \theta_{2}$. Quite naturally, I have to check $v_{1}^{* *}\left(\bar{\theta}_{1}\right)>\bar{\theta}_{2}$ due 
to $\theta_{1} \geq \theta_{2}$ :

$$
\begin{gathered}
v_{1}^{* *}\left(\bar{\theta}_{1}\right)>\bar{\theta}_{2} \Leftrightarrow \\
R<\frac{2\left(-2 q+q \sqrt{1+2 r}+\sqrt{-2 q^{2}-3 q^{2} r+2 q^{2} \sqrt{1+2 r}+q^{2}(1+2 r}\right)}{3 q^{3}} .
\end{gathered}
$$

This threshold differs only slightly from $\underline{R}$. Moreover, I have the following order:

$$
\frac{2\left(-2 q+q \sqrt{1+2 r}+\sqrt{-2 q^{2}-3 q^{2} r+2 q^{2} \sqrt{1+2 r}+q^{2}(1+2 r}\right)}{3 q^{3}}<\underline{R} .
$$

Hence, a syndicate is stable

- if $R<\underline{R}$ and condition (15) holds,

- if $R \geq \underline{R}$.

Otherwise the syndicate is not stable.

Consider Figure 3, it summarizes the results depending on the expected cash flow.

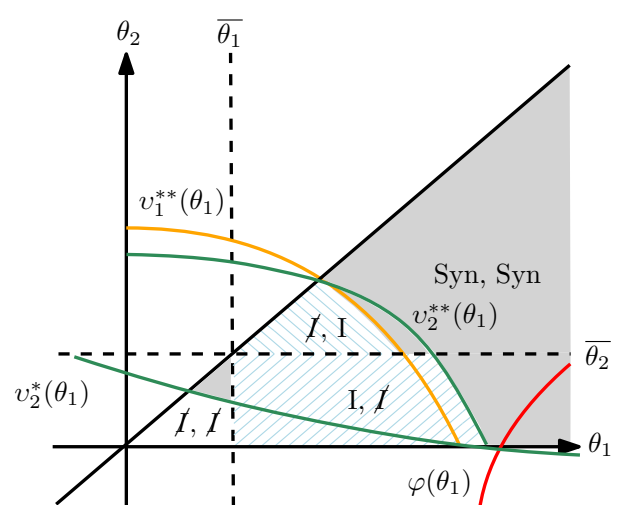

(a)

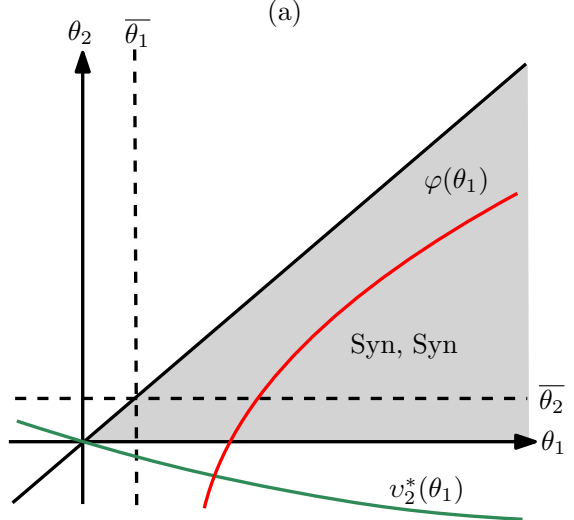

(c)

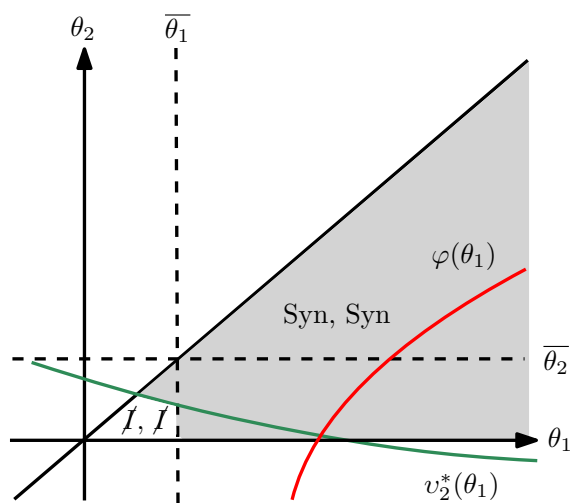

(b)

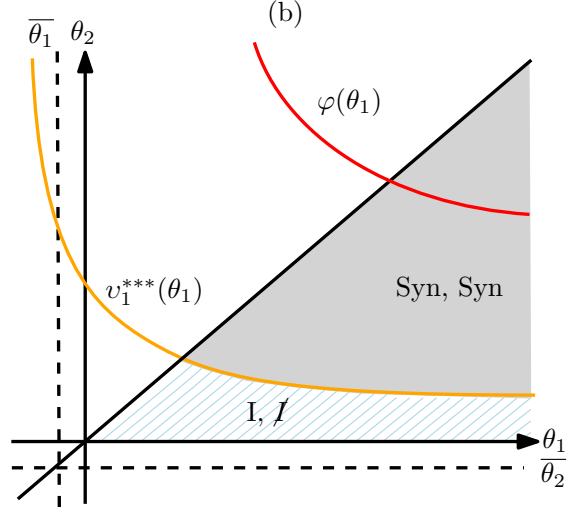

(d)

Figure 3: For Illustration (a), $r<R<\underline{R}$, for (b) $\underline{R} \leq R<\widetilde{R}$, for (c) $\widetilde{R} \leq R<\bar{R}$ and for (d) $R \geq \bar{R}$ applies. 
Proof of Proposition 5. I relax the assumption that the venture is a complement for both investors and suppose that $C V C_{2}$ is confronted with a substitute $\left(\theta_{2}<0\right)$. Furthermore, the venture is a complement for $C V C_{1}$.

(i) First, suppose that condition (8) does not hold, i.e. the equilibrium of the stand-alone setting is characterized by no investment. I have only to consider $v_{2}^{*}\left(\theta_{1}\right)$ [See, proof of Proposition 3]. The syndicate is stable if $R \geq \underline{R}$ and condition (12) holds. Otherwise the syndicate is not stable.

(ii) Suppose that condition (8) holds, i.e. the equilibrium of the stand-alone setting is characterized by a stand-alone investment of $C V C_{1}$. I consider $v_{1}^{* * *}\left(\theta_{1}\right)$ and $v_{2}^{* *}\left(\theta_{1}\right)$. Then a syndicate is stable

- if $R<\underline{R}$ and conditions (13) and (14) hold,

- if $\underline{R} \leq R<\bar{R}$ and condition (13) holds.

Otherwise the syndicate is not stable.

\section{Proof of Proposition 6.}

Intuitively, a syndicate lead to a higher value added than a stand-alone investor if the sum of the syndicate support is higher than the support of a possible stand-alone investor:

$$
s_{i}^{s y n}+s_{j}^{s y n}>\widehat{s}_{i} \Leftrightarrow \theta_{i}+\theta_{j}>\theta_{i} .
$$

If $\theta_{i} \geq 0$, then condition (31) is fulfilled only if the venture is a complement for both CVCs. Otherwise, syndicates lead to a lower value added than a stand-alone investment of the complementary $C V C_{i}$. If $\theta_{i}<0$, then condition (31) is only fulfilled if the venture is a complement for the other CVC. However, I show with Proposition (4) that the last investment pattern cannot occur.

Proof of Proposition \%. Henceforth, I show that a syndicate is stable, if $C V C_{1}$ provides nonmonetary support $\left(s_{1}^{s y n}>0\right)$ and $C V C_{2}$ sabotages the development of the venture $\left(s_{2}^{\text {syn }}<0\right)$. It is straightforward to show that $s_{2}^{s y n}<0$ if $\theta_{2}<-\frac{1}{2} R$. Moreover, one checks easily that $\frac{\partial v_{1}^{* * *}}{\partial R}>0$ and $\frac{\partial v_{2}^{* *}}{\partial R}<0$. First, I consider the 
following equation:

$$
\begin{gathered}
v_{1}^{* * *}\left(\theta_{1}\right)=-\frac{1}{2} R \\
R=\frac{r}{q \sqrt{1+2 r}}
\end{gathered}
$$

If $R<\frac{r}{q \sqrt{1+2 r}}$, then $C V C_{1}$ accepts a syndicate, whereas a possible syndicate member $C V C_{2}$ sabotages the development of the venture. Now, I check if $C V C_{2}$ is also better-off with a syndicate. I consider the following equation:

$$
v_{2}^{* *}\left(\theta_{1}\right)=v_{1}^{* * *}\left(\theta_{1}\right)=-\frac{1}{2} R \Leftrightarrow R=0
$$

Therefore, the quantity $\frac{r}{q \sqrt{1+2 r}}$ is the upper and the outside option $r$ is the lower threshold for a stable syndicate, where $C V C_{2}$ sabotages the development of the venture. Consider Figure 4, it describes the proof of Proposition 4 and 5.

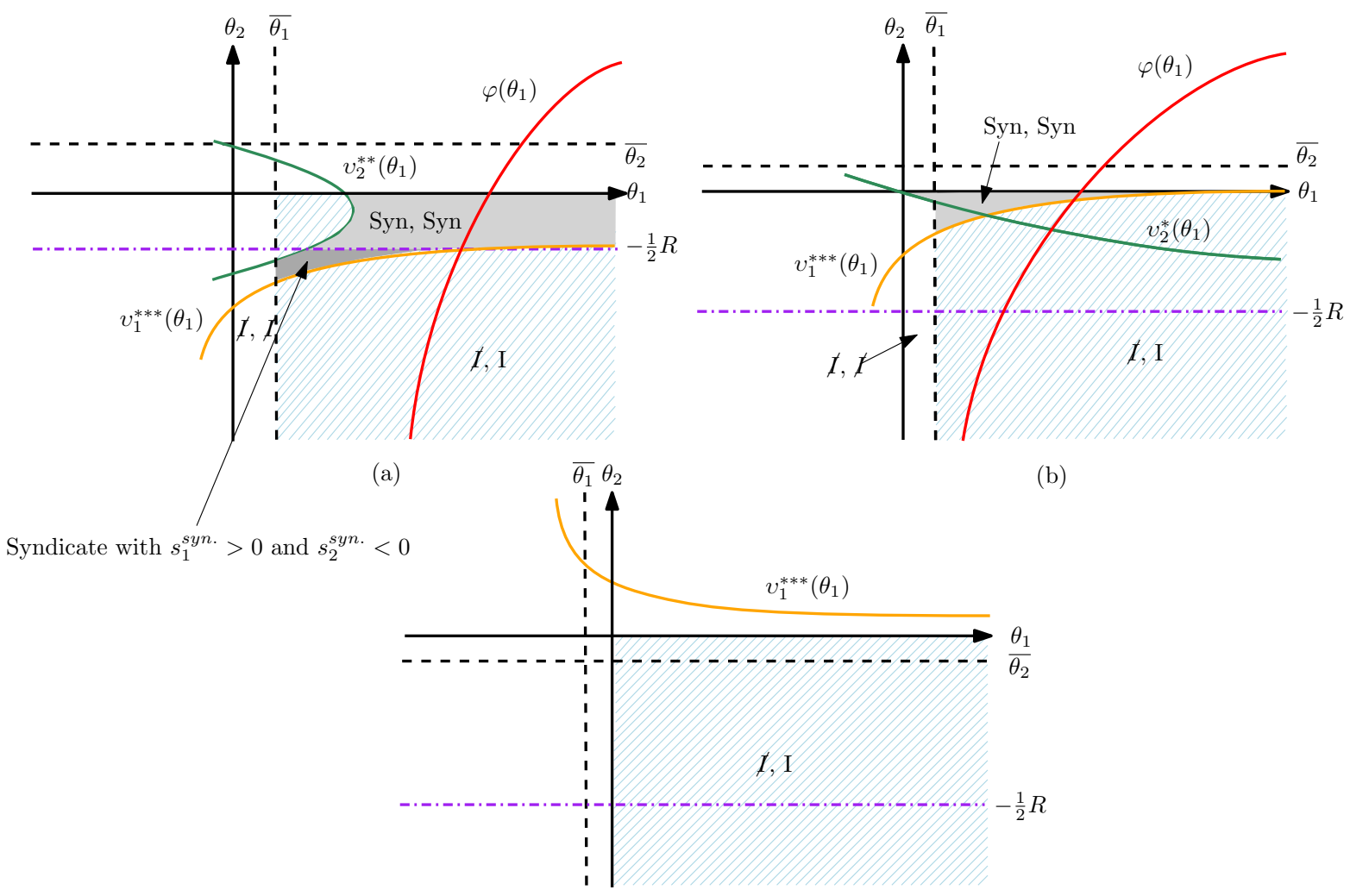

(c)

Figure 4: For Illustration (a), $r<R<\underline{R}$, for (b) $\underline{R} \leq R<\bar{R}$ and for (c) $R \geq \bar{R}$ applies. 
Proof of Proposition 8. I characterize different thresholds for the expected cash flow:

- Start with the case where the equilibrium of the stand-alone setting is characterized by no investment:

$$
\begin{aligned}
& \underline{\alpha}_{i}^{n o}<0 \text { and } \bar{\alpha}_{i}^{n o}>1 \Leftrightarrow R \geq \frac{1}{q}\left(\sqrt{2 r+\left(1+q \theta_{1}\right)^{2}}-1-g\left(\theta_{1}+\theta_{2}\right)\right) \equiv R>R_{2}^{n o}, \\
& \underline{\alpha}_{1}^{n o}<0, \underline{\alpha}_{2}^{n o} \geq 0 \text { and } \bar{\alpha}_{i}^{n o}>1 \Leftrightarrow \frac{1}{q}\left(\sqrt{2 r+\left(1+q \theta_{2}\right)^{2}}-1-g\left(\theta_{1}+\theta_{2}\right)\right)<R \\
& \leq R_{2}^{n o} \equiv R_{1}^{n o}<R \leq R_{2}^{n o}, \\
& \underline{\alpha}_{1}^{n o}<0, \underline{\alpha}_{2}^{n o} \geq 0, \bar{\alpha}_{1}^{n o} \leq 1 \text { and } \bar{\alpha}_{2}^{n o}>1 \Leftrightarrow \equiv \widehat{R}^{n o}<R \leq R_{1}^{n o} .
\end{aligned}
$$

Note that $\underline{\alpha}_{2}^{n o} \geq \bar{\alpha}_{1}^{n o} \Leftrightarrow R \leq R^{n o}$. For clarity, I skip the value of $\widehat{R}^{n o}$. This threshold is available from the author upon request.

- I proceed with the case where the equilibrium of the stand-alone setting is characterized by a stand-alone investment of $C V C_{1}$ :

$$
\begin{aligned}
& \underline{\alpha}_{1}^{1} \geq 0, \underline{\alpha}_{2}^{1}<0 \text { and } \bar{\alpha}_{i}^{1}>1 \Leftrightarrow R>\frac{1}{q}\left(\sqrt{1+2 r+2 q^{2} \theta_{1} \theta_{2}}-1\right) \equiv R>R_{1}^{1} \\
& \underline{\alpha}_{i}^{1}<0 \text { and } \bar{\alpha}_{i}^{1}>1 \Leftrightarrow-\frac{1}{q}-\theta_{1}+\sqrt{\frac{1+2 r}{q^{2}}+\frac{2 \theta_{1}}{q}+\theta_{1}^{2}-\theta_{2}^{2}}< \\
& R \leq R_{1}^{1} \equiv R_{2}^{1}<R \leq R_{1}^{1} \\
& \underline{\alpha}_{1}^{1}<0, \underline{\alpha}_{2}^{1} \geq 0 \text { and } \underline{\alpha}_{i}^{2}<0 \Leftrightarrow-\frac{1}{q}-\theta_{1}+\sqrt{\frac{1+2 r}{q^{2}}+\frac{2 \theta_{1}}{q}+\theta_{1}^{2}-\theta_{2}^{2}} \leq R \\
& \equiv R \leq R_{2}^{1}
\end{aligned}
$$

- I proceed with the case where the equilibrium of the stand-alone setting is characterized by a stand-alone investment of $C V C_{2}$ :

$$
\begin{aligned}
& \underline{\alpha}_{i}^{2}<0, \bar{\alpha}_{1}^{2}>1 \text { and } \bar{\alpha}_{2}^{2} \leq 1 \Leftrightarrow R>\frac{1}{q}\left(\sqrt{1+2 r+2 q^{2} \theta_{1} \theta_{2}}-1\right) \equiv R>R_{2}^{2} \\
& \underline{\alpha}_{i}^{2}<0 \text { and } \bar{\alpha}_{i}^{2}>1 \Leftrightarrow-\frac{1}{q}-\theta_{1}+\sqrt{\frac{1+2 r}{q^{2}}+\frac{2 \theta_{1}}{q}+\theta_{1}^{2}-\theta_{2}^{2}}<R \\
& <R_{2}^{2} \equiv R_{1}^{2}<R \leq R_{2}^{2} \\
& \underline{\alpha}_{i}^{2}>0, \bar{\alpha}_{2}^{2}>1 \text { and } \bar{\alpha}_{2}^{1} \leq 1 \Leftrightarrow R \leq R_{1}^{2}
\end{aligned}
$$

One checks easily that the different thresholds for the expected cash flow have the following order: $\widehat{R}^{n o}<R_{1}^{n o}<R_{2}^{n o}, R_{1}^{1}>R_{1}^{2}$ and $R_{2}^{1}<R_{2}^{2}$. 
Proof of Proposition 9. The proof of this proposition is straightforward. I have already shown the expected cash flow benchmarks (e.g. condition (19) up to (22)) and the derivations $\frac{\partial \widetilde{U}_{1}^{s y n}}{\partial \alpha}$ and $\frac{\partial \widetilde{U}_{2}^{s y n}}{\partial \alpha}$, respectively. Hence, I only state the following condition to complete the proof:

$$
\alpha_{1}^{p e} \geq \alpha_{2}^{p e} \Leftrightarrow R \leq \frac{-2 q-q^{2}\left(\theta_{1}+\theta_{2}\right)+\sqrt{q^{2}\left(8 r+\left(2+q\left(\theta_{1}+\theta_{2}\right)^{2}\right.\right.}}{2 q^{2}} .
$$

If condition (32), (20) and (22) do not hold, then every shareholding $\alpha \in\left[\alpha_{2}^{p e} ; \alpha_{1}^{p e},\right]$ is pareto efficient. Otherwise, if condition (32) does not hold, then every shareholding $\alpha \in\left[\alpha_{1}^{p e} ; \alpha_{2}^{p e},\right]$ is pareto efficient.

\section{Proof of Proposition 11.}

(i) I have already shown the calculation of the shareholding if the disagreement point is characterized by no investment. Hence, I check if the Nash bargaining solution can implement the shareholding $\alpha^{\max }$ :

$$
\frac{-2 r+g\left(R-\theta_{1}+\theta_{2}\right)\left(2+q 8 R+\theta_{1}+\theta_{2}\right)}{2\left(-2 r+q R\left(2+q\left(R+\theta_{1}+\theta_{2}\right)\right)\right)}=\frac{1}{2}+\frac{\theta_{2}-\theta_{1}}{2 R} \Leftrightarrow \theta_{1}=\theta_{2}
$$

(ii) Suppose the disagreement point is characterized by an investment of $C V C_{1}$. Then the following utility difference exist: $\Delta U_{1}^{1}=\delta U^{\text {joint }}-U_{1}^{1}$ and $\Delta U_{2}^{1}=$ $(1-\delta) U^{\text {joint }}-U_{2}^{1}$. The Nash bargaining solution can be determined for $C V C_{1}$ through the following maximization problem:

$$
\widetilde{\delta}=\underset{\delta \in[0 ; 1]}{\operatorname{argmax}}\left\{\Delta U_{1}^{1} \cdot \Delta U_{2}^{1}\right\}
$$

This yields:

$$
\widetilde{\delta}=\frac{-4+4 q\left(R+\theta_{1}\right)+q^{2}\left(R^{2}\left(2+2 \alpha-2 \alpha^{2}\right)+E\right.}{2\left(-4+2 r+2 q\left(R+\theta_{1}+\theta_{2}\right)+q^{2}\left(R^{2}\left(1+2 \alpha-2 \alpha^{2}\right)+F\right.\right.},
$$

where

$$
\begin{aligned}
& \left.E=2 \theta_{1}^{2}+2 \theta_{1} \theta_{2}+\theta_{2}^{2}+R\left(6 \theta_{1}-2 \alpha \theta_{1}+2 \alpha \theta_{1}\right)\right), \\
& F=\theta_{1}^{2}+4 \theta_{1} \theta_{2}+\theta_{2}^{2}+2 R\left(2 \theta_{1}-\alpha \theta_{1}+\theta_{2}+\alpha \theta_{2} .\right.
\end{aligned}
$$


I have to determine the particular share $\alpha$ of the expected cash flow:

$$
\widetilde{U}_{1}^{\text {syn }}=\widetilde{\delta} U^{\text {joint }} \Leftrightarrow \alpha=\frac{-4 r+q\left(2 q R^{2}+2 R\left(2+q \theta_{1}\right)+q \theta_{2}\left(\theta_{2}-2 \theta_{1}\right)\right)}{2\left(-2 r+q R\left(2+q\left(R+\theta_{1}+\theta_{2}\right)\right)\right)}
$$

Next, I justify the share $\alpha^{\max }$ that maximizes the joint surplus of a syndicate by means of bargaining:

$$
\begin{aligned}
& \frac{-4 r+q\left(2 q R^{2}+2 R\left(2+q \theta_{1}\right)+q \theta_{2}\left(\theta_{2}-2 \theta_{1}\right)\right)}{2\left(-2 r+q R\left(2+q\left(R+\theta_{1}+\theta_{2}\right)\right)\right)}=\frac{1}{2}+\frac{\theta_{2}-\theta_{1}}{2 R} \Leftrightarrow \\
& \theta_{1}=\frac{\left.r-q R+q^{2} R\left(-R+\theta_{2}\right)+\sqrt{r^{2}-2 q r R+q^{2} R^{2}\left(1+q^{2} \theta_{2}^{2}\right.}\right)}{q^{2} R}
\end{aligned}
$$

(iii) Suppose the disagreement point is characterized by an investment of $C V C_{2}$. The same approach holds as for part (ii). Thus, I only show the particular shareholding:

$$
\widetilde{U}_{1}^{\text {syn }}=\check{\delta} U^{\text {joint }} \Leftrightarrow \alpha=\frac{-q^{2} \theta_{1}\left(-2 R+\theta_{1}-2 \theta_{2}\right)}{2\left(-2 r+q R\left(2+q\left(R+\theta_{1}+\theta_{2}\right)\right)\right)}
$$

Last, I justify the share $\alpha^{\max }$ that maximizes the joint surplus of a syndicate by means of bargaining:

$$
\begin{aligned}
& \frac{-q^{2} \theta_{1}\left(-2 R+\theta_{1}-2 \theta_{2}\right)}{2\left(-2 r+q R\left(2+q\left(R+\theta_{1}+\theta_{2}\right)\right)\right)}=\frac{1}{2}+\frac{\theta_{2}-\theta_{1}}{2 R} \Leftrightarrow \\
& \theta_{1}=\frac{R+\theta_{2}\left(-2 r+q R\left(2+q\left(R+\theta_{2}\right)\right)\right)}{2\left(-r+q R\left(1+q\left(R+\theta_{2}\right)\right)\right)}
\end{aligned}
$$

\section{References}

Bayar, O. and Chemmanur, T. J. (2011). Ipos versus acquisitions and the valuation premium puzzle: A theory of exit choice by entrepreneurs and venture capitalists, Journal of Financial and Quantitative Analysis 46(06): 1755-1793.

Benson, D. and Ziedonis, R. H. (2009). Corporate venture capital as a window on new technologies: Implications for the performance of corporate investors when acquiring startups, Organization Science 20(2): 329-351.

Block, Z. and MacMillan, I. C. (1993). Corporate Venturing: Creating New Businesses Within the Firm: Creating new businesses within the firm, Harvard Business School Press and Beard Books, Washington, D.C. 
Brander, J. A., Amit, R. and Antweiler, W. (2002). Venture-capital syndication: Improved venture selection vs. the value-added hypothesis: Improved venture selection vs. the value-added hypothesis, Journal of Economics \& Management Strategy 11(3): 423-452.

Casamatta, C. and Haritchabalet, C. (2007). Experience, screening and syndication in venture capital investments, Journal of Financial Intermediation 16(3): 368398.

Cestone, G., Lerner, J. and White, L. (2006). The design of syndicates in venture capital.

Chemmanur, T. J. and Loutskina, E. (2008). How do corporate venture capitalists create value for entrepreneurial firms?

Chesbrough, H. and Tucci, C. L. (2002). Corporate venture capital in the context of corporate innovation.

Dushnitsky, G. (2008). Corporate venture capital: Past evidence and future directions, in M. Casson, A. Basu, N. Wadeson and B. Yeung (eds), The Oxford Handbook of Entrepreneurship, Oxford Handbooks Ser, Oxford University Press, Incorporated, New York, pp. 387-431.

Dushnitsky, G. (2012). Corporate venture capital in the twenty-first century: an integral part of firms' innovation toolkit, in D. Cumming (ed.), The Oxford Handbook of Venture Capital, Oxford University Press, New York, pp. 211-245.

Dushnitsky, G. and Shaver, J. M. (2009). Limitations to interorganizational knowledge acquisition: The paradox of corporate venture capital, Strategic Management Journal 30(10): 1045-1064.

Fluck, Z., Garrison, K. and Myers, S. (2005). Venture capital contracting and syndication: An experiment in computational corporate finance.

Gompers, P. and Lerner, J. (2000). The determinants of corporate venture capital success: Organizational structure, incentives, and complementarities, in R. Morck (ed.), Concentrated corporate ownership, A National Bureau of Economic Research conference report, University of Chicago Press, Chicago, pp. 17-54.

Hellmann, T. (2002). A theory of strategic venture investing, Journal of Financial Economics 64(2): 285-314. 
Hill, S. A., Maula, M. V. J., Birkinshaw, J. M. and Murray, G. C. (2009). Transferability of the venture capital model to the corporate context: Implications for the performance of corporate venture units, Strategic Entrepreneurship Journal 3(1): 3-27.

Hochberg, Y. V., Ljungqvist, A. and Lu, Y. (2007). Whom you know matters: Venture capital networks and investment performance: Venture capital networks and investment performance, The Journal of Finance 62(1): 251-301.

Ivanov, V. I. and Xie, F. (2010). Do corporate venture capitalists add value to start-up firms? evidence from ipos and acquisitions of vc-backed companies: Evidence from ipos and acquisitions of vc-backed companies, Financial management 39(1): 129-152.

Laengle, S. and Loyola, G. (2012). Bargaining and negative externalities, Optimization Letters 6(3): 421-430.

Lerner, J. (1994). The syndication of venture capital investments, Financial management 23(3): 16-27.

MacMillan, I., Roberts, E., Livada, V. and Wang, A. (2012). Corporate Venture Capital (CVC) Seeking Innovation and Strategic Growth: Recent Patterns in CVC Mission, Structure and Investment, CreateSpace Independent Publishing Platform.

Masulis, R. W. and Nahata, R. (2009). Financial contracting with strategic investors: Evidence from corporate venture capital backed ipos, Journal of Financial Intermediation 18(4): 599-631.

Maula, M. V. J. (2001). Corporate Venture Capital and the Value-Added for Technology Based New Firms, PhD thesis, Helsinki University of Technology, Espoo.

McNally, K. (1997). Corporate venture capital: Bridging the equity gap in the small business sector, Vol. 2 of Routledge studies in small business, Routledge, London and New York.

National Venture Capital Association (2016). 2016-Q2-Corporate-Venture-CapitalAnalysis.

URL: www.nvca.org/?ddownload=37r2

Park, H. D. and Steensma, H. K. (2012). When does corporate venture capital add value for new ventures?, Strategic Management Journal 33(1): 1-22. 
Riyanto, Y. E. and Schwienbacher, A. (2006). The strategic use of corporate venture financing for securing demand, Journal of Banking \& Finance 30(10): 2809-2833.

Sah, R. S. and Stiglitz, J. E. (1986). The architecture of economic systems, American Economic Review 76(4): 716-727.

Sharifzadeh, A. and Walz, U. (2012). Syndication of vc investments, governance and contract design, in D. Cumming (ed.), The Oxford Handbook of Venture Capital, Oxford University Press, New York.

Sørensen, M. (2007). How smart is smart money? a two-sided matching model of venture capital, The Journal of Finance 62(6): 2725-2762.

Souitaris, V. and Zerbinati, S. (2014). How do corporate venture capitalists do deals? an exploration of corporate investment practices, Strategic Entrepreneurship Journal 8(4): 321-348.

Tian, X. (2011). The role of venture capital syndication in value creation for entrepreneurial firms, Review of Finance 16(1): 245-283.

Winters, T. E. and Murfin, D. L. (1988). Venture capital investing for corporate development objectives, Journal of Business Venturing 3(3): 207-222.

Yung, C. (2012). Venture capital before the first dollar, in D. Cumming (ed.), The Oxford Handbook of Venture Capital, Oxford University Press, New York. 